ARTICLE

\title{
Pantothenate biosynthesis is critical for chronic infection by the neurotropic parasite Toxoplasma gondii
}

\author{
Matteo Lunghi (iD 1,5, Joachim Kloehn (10) 1,5, Aarti Krishnan (iD 1, Emmanuel Varesio (10 2,3, Oscar Vadas (i) ${ }^{1,4}$ \& \\ Dominique Soldati-Favre (iD ${ }^{1 凶}$
}

Coenzyme $\mathrm{A}(\mathrm{COA})$ is an essential molecule acting in metabolism, post-translational modification, and regulation of gene expression. While all organisms synthesize CoA, many, including humans, are unable to produce its precursor, pantothenate. Intriguingly, like most plants, fungi and bacteria, parasites of the coccidian subgroup of Apicomplexa, including the human pathogen Toxoplasma gondii, possess all the enzymes required for de novo synthesis of pantothenate. Here, the importance of $\mathrm{CoA}$ and pantothenate biosynthesis for the acute and chronic stages of $T$. gondii infection is dissected through genetic, biochemical and metabolomic approaches, revealing that CoA synthesis is essential for T. gondii tachyzoites, due to the parasite's inability to salvage CoA or intermediates of the pathway. In contrast, pantothenate synthesis is only partially active in $T$. gondii tachyzoites, making the parasite reliant on its uptake. However, pantothenate synthesis is crucial for the establishment of chronic infection, offering a promising target for intervention against the persistent stage of

T. gondii.

\footnotetext{
${ }^{1}$ Department of Microbiology and Molecular Medicine, University of Geneva, CMU, Rue Michel-Servet 1, 1211 Geneva, Switzerland. ${ }^{2}$ Institute of Pharmaceutical Sciences of Western Switzerland (ISPSO), University of Geneva, CMU, Rue Michel-Servet 1, 1211 Geneva, Switzerland. ${ }^{3}$ Mass Spectrometry Core Facility (MZ 2.0), University of Geneva, 1211 Geneva, Switzerland. ${ }^{4}$ Protein and peptide purification platform, University of Geneva, CMU, Rue Michel-

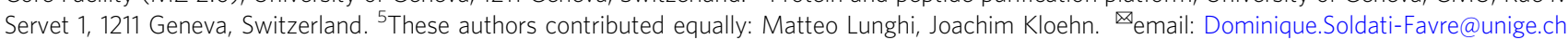


T he Apicomplexa phylum comprises thousands of parasites that infect humans and animals, such as Plasmodium spp., the causative agents of malaria, and Cryptosporidium spp. responsible for diarrheal disease. Toxoplasma gondii is the most ubiquitous member of the phylum, infecting all warm-blooded animals including an estimated third of the human population ${ }^{1}$. Infection typically occurs through the accidental intake of oocysts from contaminated food and water, or consumption of tissue cysts from infected meat. Primary infection during pregnancy can cause miscarriage or stillbirth following placental infection of the fetus ${ }^{2}$. During an effective immune response, the fast replicating tachyzoites are cleared, while some parasites convert into slow growing bradyzoites that persist within cysts, predominantly in the brain and muscle tissues, for the lifetime of the host $^{3}$. This chronic infection is generally asymptomatic but poses a severe risk of toxoplasmosis recrudescence in case of immunosuppression ${ }^{4,5}$. Clinically available drugs are effective against tachyzoites, but fail to eradicate the encysted, quasiquiescent bradyzoites.

As an obligate intracellular parasite, T. gondii and other apicomplexans rely on the uptake of essential nutrients from their host, as well as on the de novo synthesis of metabolites which cannot be sufficiently salvaged. The metabolic needs and capabilities of $T$. gondii bradyzoites are poorly characterized due to the technical challenges associated with studying this parasite state, particularly in its natural niche. The identification of salvaged metabolites or synthesis pathways that are essential for the establishment of chronic stage is a critical step towards the treatment of chronic toxoplasmosis.

Coenzyme A ( $\mathrm{CoA})$ is a ubiquitous and essential hub metabolite found in all organisms, acting in gene regulation, posttranslational protein modification and several metabolic pathways, including the tricarboxylic acid (TCA) cycle as well as heme and fatty acid synthesis. In T. gondii, the most abundant CoA-derivative, acetyl-CoA, is produced by four different enzymes, each of which localizes to one of three metabolically active subcellular compartments: the mitochondrion, the cytosol/ nucleus, and the apicoplast, a relict plastid organelle found in many apicomplexans ${ }^{6-8}$. Aside from acetyl-CoA, synthesis of other critical metabolites such as malonyl-CoA, succinyl-CoA and acyl-CoAs also depend on CoA availability. The synthesis of $\mathrm{CoA}$ involves five conserved enzymatic steps and uses pantothenate (vitamin $\mathrm{B}_{5}$, Pan) as a precursor. Mining of the $T$. gondii genome confirmed the presence of a complete pathway for CoA biosynthesis, including the previously unannotated gene for the dephospho-CoA kinase (DPCK) $)^{9,10}$. The pathway is conserved in all apicomplexans (Fig. 1a, b, Supplementary Data 1). Intriguingly, unlike their human and animal hosts, T. gondii and other coccidians also possess the genes encoding enzymes to synthesize the CoA precursor Pan (Fig. 1a, b, Supplementary Data 1). Here, we scrutinized the parasite's ability to synthesize and/or salvage intermediates of the Pan/CoA pathway and the importance of several biosynthesis steps for the clinically relevant life cycle stages of $T$. gondii. We uncover that CoA synthesis is essential in T. gondii, while Pan synthesis is dispensable in tachyzoites due to an efficient salvage pathway. Remarkably, Pan synthesis becomes critical during chronic infection, with disruption of the pathway resulting in a marked reduction in cyst numbers. These findings are invaluable for the development of much needed drugs to treat acute and chronic toxoplasmosis.

\section{Results and discussion}

Initiation of CoA biosynthesis from Pan is essential for Toxoplasma gondii and relies on a heteromeric PanK-complex. In order to probe the importance of CoA synthesis in T. gondii, the role of several putative enzymes of the pathway was characterized, namely, two pantothenate kinases (PanK1 +2$)$, the phosphopantothenoylcysteine decarboxylase (PPCDC) and DPCK (Fig. 2a), catalyzing the first, an intermediate and the last step of CoA synthesis, respectively. The initial enzymatic step consists in the phosphorylation of Pan to form $4^{\prime}$-phosphopantothenate (PPan) and is catalyzed by PanKs ${ }^{9,11}$. T. gondii, as most apicomplexans, possesses two PanK genes: PanK1 (TGME49_307770) and PanK2 (TGME49_235478) (nomenclature based on sequence similarity and existing literature $\left.{ }^{11}\right)$. To examine the function of the PanKs, both proteins were C-terminally tagged with haemaglutinin (HA-) epitope and fused to a mini auxin degron-haemagglutinin-tag (mAID-HA, Supplementary Fig. 1a) in an RH ku80-ko hxgprt-ko::Tir1 strain (Tir1 parental) at the endogenous locus by CRISPR/Cas9 editing ${ }^{12,13}$. PanK1-mAID-HA and PanK2-mAID-HA migrated close to the predicted molecular weight (MW) of $144 \mathrm{kDa}$ and $190 \mathrm{kDa}$, respectively, and were efficiently downregulated upon addition of auxin (indole-3-acetic acid, IAA) ${ }^{14}$ as shown by western blot (Fig. 2b)

Both enzymes were localized by immunofluorescence assays (IFAs), with PanK1 and PanK2-mAID-HA tagged strains presenting a faint, dotty cytoplasmic staining (Fig. 2c). Addition of IAA to the culture medium over $24 \mathrm{~h}$ growth of the parasite resulted in a marked loss of the dotty signal in the two strains (Fig. 2c), confirming efficient downregulation of PanK1 and PanK2 as observed by western blot (Fig. 2b). Crucially, downregulation of either PanK1 or PanK2 over $24 \mathrm{~h}$ was accompanied by severe loss of parasite morphology, as seen by staining with actin (Fig. 2c) and GAP45, a marker of the parasite pellicle (Fig. 2d).

As expected, given the severe morphological defects after only $24 \mathrm{~h}$ of PanK1 or PanK2 downregulation, PanK1-mAID-HA and PanK2-mAID-HA parasites failed to form lysis plaques in host cell monolayers (human foreskin fibroblasts, HFFs) in the presence of IAA over 7 days (Fig. 2e), quantified in Fig. 2f. These findings reveal that PanK1 and PanK2 are individually essential for the parasite's lytic cycle.

The fitness conferring nature of PanK1 and PanK2 is consistent with data from a genome-wide fitness screen, which assigned a highly negative fitness score to both enzymes (Fig. 1a) $)^{15}$ and led us to hypothesize that the enzymes are not functionally redundant but instead both enzymes may be required to form a functional PanK-complex. In Escherichia coli, PanKs have been shown to form homodimers ${ }^{16}$, although heteromeric PanK complexes have not been reported until recently ${ }^{17}$. Coimmunoprecipitation experiments of dually tagged strains PanK1-mAID-HA/PanK2-Ty and PanK2-mAID-HA/PanK1-Ty revealed that PanK1 and PanK2 form a heteromeric complex, causing PanK1 to be pulled down with PanK2 and vice versa (Fig. 2g). These results are consistent with a recent study reporting that $P$. falciparum and T. gondii PanKs function in a heteromeric PanK complex ${ }^{17}$. Of relevance, IAA-induced destabilization of either T. gondii PanK1 or PanK2 did not lead to the degradation of the other PanK (Supplementary Fig. 1b). Given that both PanKs are required to form an active heteromeric complex, the following phenotypical characterization was carried out focusing on parasites depleted in PanK1 only.

To identify how quickly upon initiation of PanKdownregulation parasite fitness deteriorates, as judged by cell morphology, additional IFAs were performed on PanK1-mAIDHA parasites treated for 0,8 and $12 \mathrm{~h}$ with IAA. The onset of cell deformation of varying types and severity was revealed, using a parasite pellicle marker, already $12 \mathrm{~h}$ after IAA-treatment. Parasites appeared bloated, likely due to failed cytokinesis (Fig. $2 \mathrm{~h} 3$, for normal morphology of non-dividing and dividing parasites, see Fig. $2 \mathrm{~h} 1$ and $2 \mathrm{~h} 2$, respectively), were distributed 
a

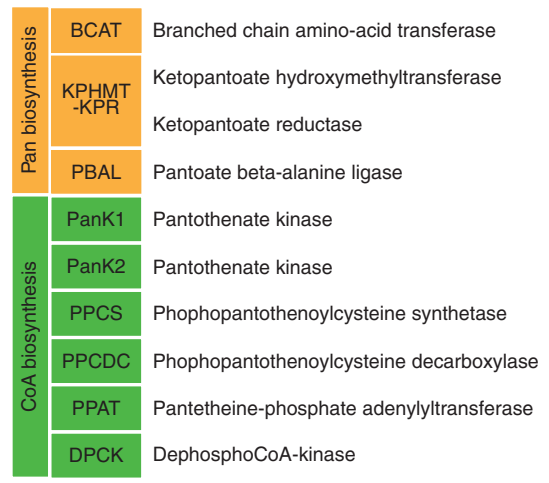

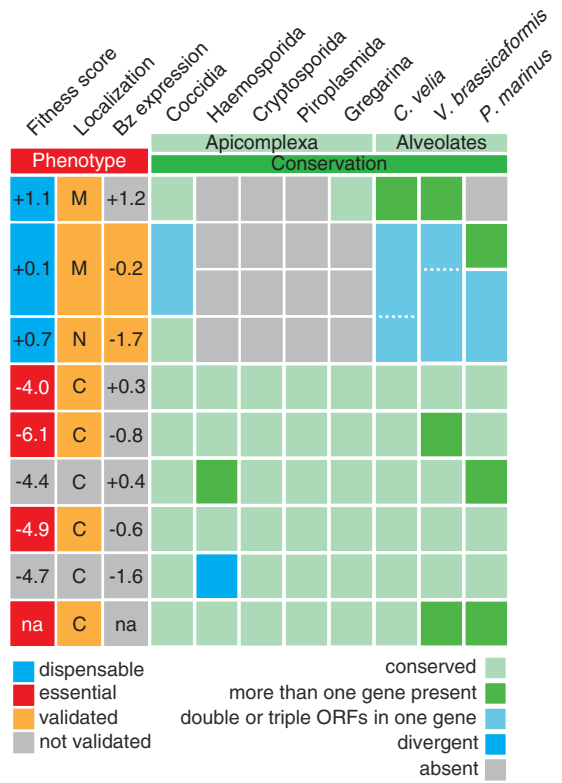

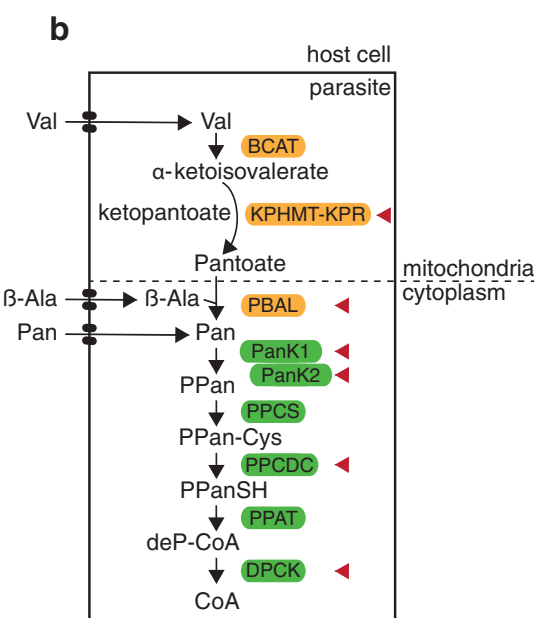

Fig. 1 Pan and CoA biosynthesis pathways in T. gondii. a Table representing the fitness cost, localization, and stage expression of enzymes for pantothenate and coenzyme A biosynthesis in T. gondii, and conservation of the genes in other alveolates. Fitness scores from a genome-wide fitness screen are given (positive values indicate dispensability, while negative values suggest essentiality) ${ }^{15}$, together with dispensability/essentiality determined from this and previous studies (blue - dispensable, red - essential). Localizations, experimentally determined in this or previous studies, are given for all enzymes involved in pantothenate ( $P a n$ ) or coenzyme $A(C o A)$ biosynthesis ( $M$ mitochondrion, $N$ nucleus, $C$ cytosol), in gray when predicted but not experimentally validated. $\log _{2}$ fold change in RNA levels from an RNA-seq analysis 75 are provided (bradyzoite (Bz) expression levels relative to tachyzoites) and complemented by protein expression levels determined in this study (orange - validated experimentally). Conservation is determined by a BLAST search (e-value $<\mathrm{E}-04$ ). Dotted lines represent the situation where single/fused genes are present, as well as one open reading frame (ORF) spanning all 3 genes. b Cartoon scheme of the Pan and CoA biosynthesis pathways. Valine (Val), $\beta$-Alanine ( $\beta$-Ala) and Pan are likely salvaged through unknown transporters. Enzymes are in boxes. Red arrowheads highlight the enzymes under scrutiny in this manuscript. Abbreviations: PPan, 4'-

phosphopantothenate; PPan-Cys, 4'-phospho-N-pantothenoyl cysteine; PPanSH, 4'-phosphopantetheine; deP-CoA, dephospho-CoA; CoA, coenzyme A.

abnormally in the vacuole (Fig. $2 \mathrm{~h} 4$ ), or displayed a complete loss of inner membrane complex (IMC) integrity (Fig. 2h5).

Additionally, employing markers for the two key metabolic organelles, which harbor metabolic pathways that critically depend on $\mathrm{CoA}$, the apicoplast and the mitochondrion, parasites displayed no aberrant organelle morphology after $8 \mathrm{~h}$ IAA (Fig. 2i, j). The absence of deformed parasites at $8 \mathrm{~h}$ IAA, while predominant at $24 \mathrm{~h}$ IAA, was quantified by an intracellular growth assay (Fig. 2k).

To examine whether the PanKs catalyze the expected reaction in CoA synthesis, targeted metabolomic analyses were performed, measuring the abundance of $\mathrm{CoA}$ and three of its precursors (Pan, $4^{\prime}$-phospho- $N$-pantothenoyl cysteine, PPan-Cys and dephospho-CoA, deP-CoA) in parasite extracts by liquid chromatography-mass spectrometry (LC-MS). Based on the above phenotyping, quantitative LC-MS analyses were performed after $8 \mathrm{~h}$ IAA treatment (Fig. 2l). Consistent with PanK1 catalyzing the phosphorylation of Pan, its downregulation led to a significant increase in its substrate, Pan. PPan-Cys was not detected in the parental line and the PanK1 depleted parasites. deP-CoA was significantly decreased in PanK1-mAID parasites upon addition of IAA compared to the parental Tirl line +IAA but was similarly reduced in PanK1-mAID parasites -IAA. Instead, CoA was significantly ( 2-fold) reduced specifically upon downregulation of PanK1 compared to its controls. The accumulation of its substrate, Pan, and reduction in the endproduct, CoA, are consistent with the expected function of PanKs.

We conclude that $T$. gondii possesses two PanKs which function in a heteromeric complex that catalyzes the phosphorylation of Pan towards CoA synthesis. Obstruction of this step causes severe morphological defects and results in parasite death.
T. gondii cannot rely on the salvage of CoA or intermediates of the CoA synthesis pathway. Following enzymatic activity of the PanKs, cysteine is added to PPan through the phosphopantothenoylcysteine synthetase (PPCS), and the subsequent decarboxylation catalyzed by PPCDC leads to the generation of 4'-phosphopantetheine (PPanSH). Critically, the function of PPCDC can be bypassed by the promiscuous activity of some PanKs, which catalyze the phosphorylation of pantetheine $(\mathrm{PanSH})^{18,19}$. In Plasmodium yoelii and Plasmodium berghei blood stages ${ }^{20,21}$, this salvage pathway was shown to be active, bypassing the CoA synthesis enzymes PPCS and PPCDC (Fig. 3a). In contrast, direct $\mathrm{PPanSH}$ salvage has been proposed in Drosophila and mammalian cell culture models and could explain dispensability of both PanKs in P. berghei ${ }^{19,22,23}$. PPanSH in culture medium can be generated by CoA hydrolysis through serum hydrolases. To determine whether either salvage pathway can contribute to CoA synthesis in T. gondii, PPCDC (TGME49_242880), the enzyme upstream of the potential PanSH salvage, was characterized.

C-terminal mAID-HA tagged PPCDC was generated (Supplementary Fig. 1c) but could not be detected by western blot, whereas a C-terminal 3xTy-tagged fusion in the endogenous locus confirmed that PPCDC-Ty migrates close to the predicted molecular weight by western blot $(34 \mathrm{kDa}$, Fig. $3 \mathrm{~b})$. By IFA, PPCDC-Ty showed a cytoplasmic and nuclear signal (Fig. 3c). The signal detected by IFA for PPCDC-mAID-HA was weak but sufficient to confirm its efficient downregulation following addition of IAA for $24 \mathrm{~h}$ (Fig. 3d). As described above for the PanKs, PPCDC downregulation led to a dramatic loss of cell morphology based on the actin staining (Fig. 3d) and pellicle marker, $24 \mathrm{~h}$ after IAA treatment (Fig. 3e). Consistent with this 
a

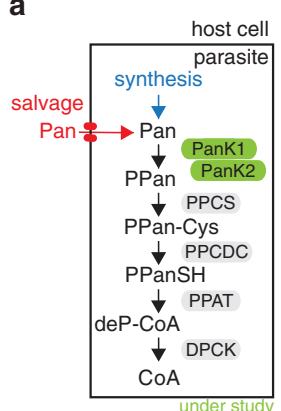

d

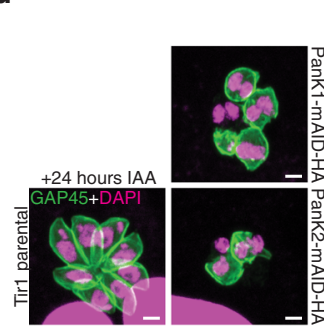

e

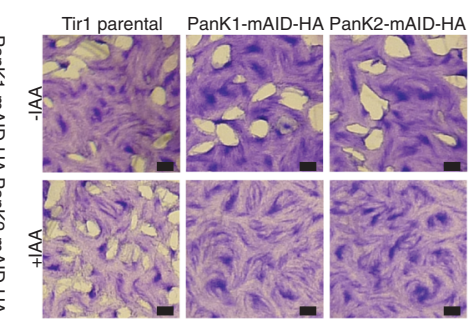

f

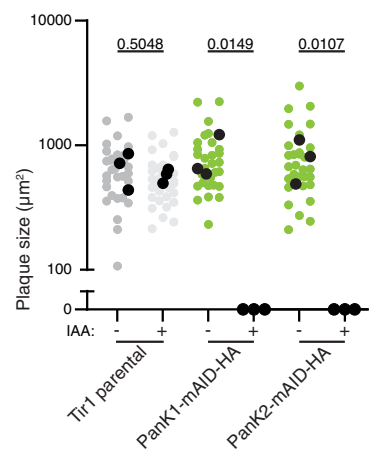

Tir1 parental PanK1-mAID-HA PanK2-mAID-HA

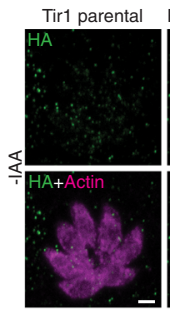

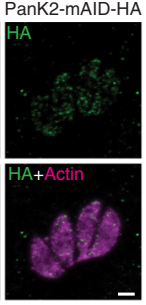

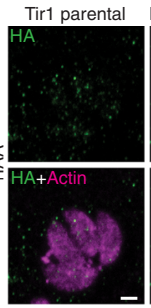

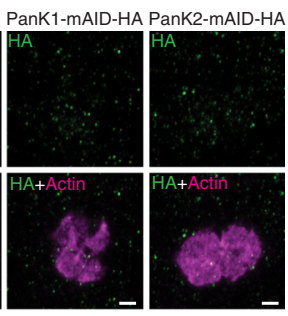

g parental

PanK2 PanK2-mAID-HA -mAID-HA PanK1-Ty
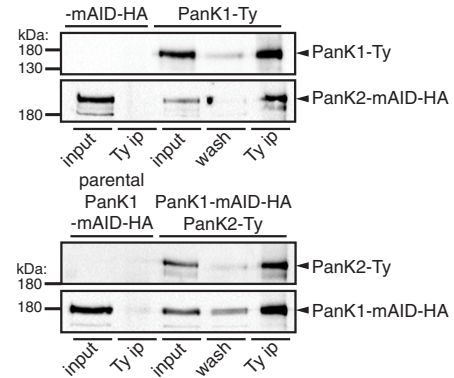

h

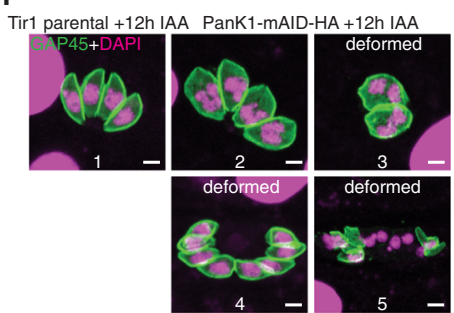

i
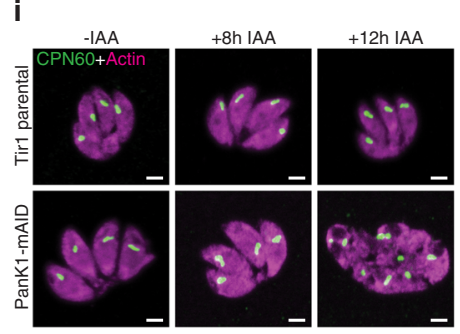

j
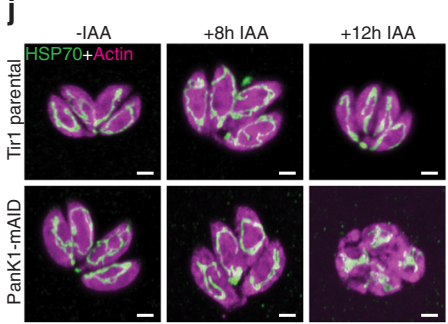

k

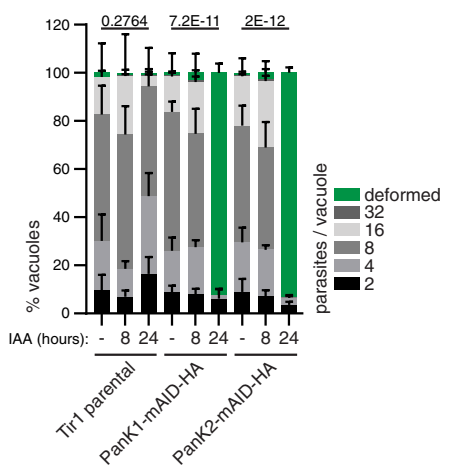

I

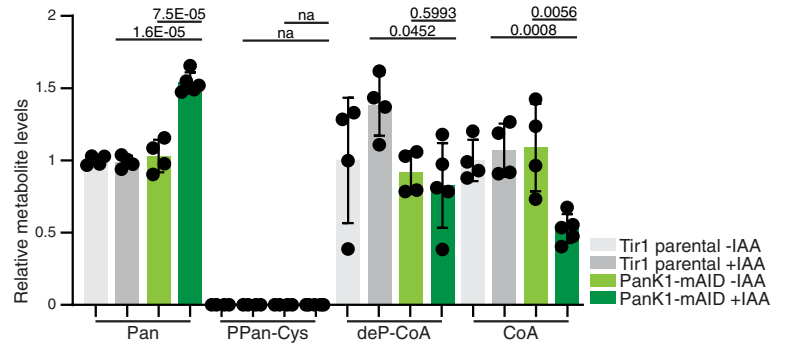

Fig. 2 Initiation of CoA biosynthesis from Pan is essential for T. gondii and relies on a heteromeric PanK-complex. a Scheme of the CoA biosynthesis pathway, highlighting the T. gondii pantothenate kinases (PanKs). See Fig. 1 for abbreviations of enzymes and metabolites. $\mathbf{b}$ Western blot of endogenous C-terminally mAID-HA tagged PanK1 and PanK2 (expected MW $144 \mathrm{kDa}$ and $190 \mathrm{kDa}$, respectively) in presence of indole-3-acetic acid (IAA). Anti HA, anti actin as loading control $(n=4)$. c Immunofluorescence assay (IFA) of PanK1-mAID-HA and PanK2-mAID-HA. Antibodies: anti HA and anti actin marking the parasite cytoplasm $(n=5)$. d IFA of $24 \mathrm{~h}$ IAA treated PanK1- and PanK2-mAID-HA. Antibodies anti GAP45 for parasite pellicle, DAPI for nuclear staining $(n=3)$. e Plaque assays of PanK1-mAID-HA and PanK2-mAID-HA in the presence of IAA $(n=4)$. f Quantification of plaque size. Black dots present the average of each independent experiment. $(n=3,2$-sided $t$-test). $\mathbf{g}$ Western blot of co-immunoprecipitation (IP) assay of dually tagged PanK1-Ty/PanK2-mAID-HA (top, $n=3$ ) and PanK1-mAID-HA/PanK2-Ty (bottom, $n=4$ ). Antibodies for IP: anti-Ty; anti-HA and anti-Ty for revelation. h IFA of PanK1-mAID-HA with IAA for $12 \mathrm{~h}$. Antibodies: anti GAP45 for parasite pellicle, DAPI for nuclear staining $(n=3)$. $\mathbf{i}, \mathbf{j}$ IFAs of apicoplasts and mitochondria, respectively, at the indicated timepoints of IAA addition in PanK1-mAID-HA. Antibodies: anti actin for parasite cytoplasm, anti CPN60 for apicoplasts, anti HSP70 for mitochondria $(n=3)$. $\mathbf{k}$ Intracellular growth assay of the PanK1- and PanK2-mAID-HA. Deformed parasites were scored as indicated in panel $\mathrm{h}(n=3-6$, mean and SD, 2 tailed $t$-test on the "deformed" category). I LC-MS analysis of indicated metabolite abundances in PanK1 depleted parasites ( $8 \mathrm{~h}+\mathrm{IAA}, n=4-5$, mean and SD, 2-sided Student's $t$ test). Equal number of parasites were analyzed, and metabolite levels normalized to an internal standard $\left({ }^{13} \mathrm{C}_{6} /{ }^{15} \mathrm{~N}\right.$-isoleucine) and quantified relative to Tir1 parental -IAA (abundance $=1$ ). $n$ number of independent biological replicates. Source data are provided as a Source Data file. White scale bar $2 \mu \mathrm{m}$, black scale bar $1 \mathrm{~mm}$. Black asterisk indicates unspecific signal. 


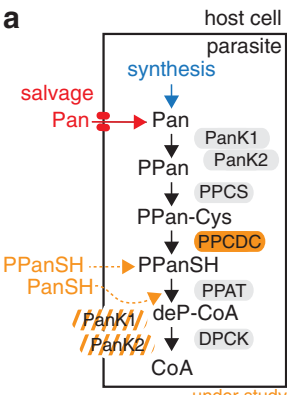

b

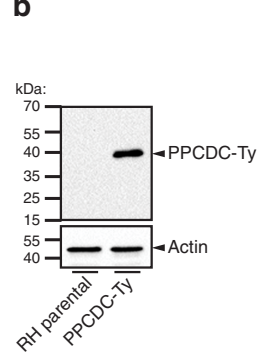

c

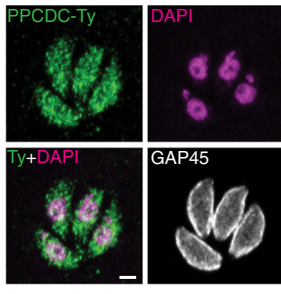

d

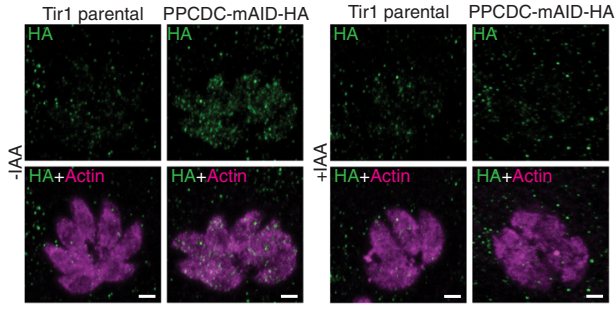

e

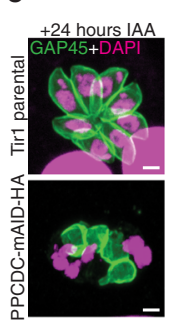

$\mathbf{f}$

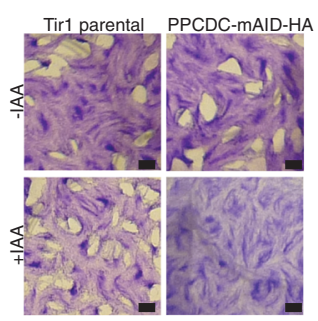

g

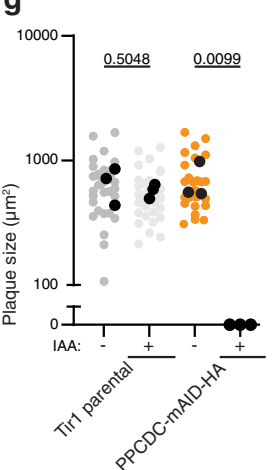

h

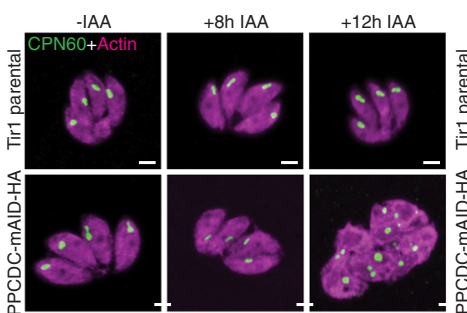

i
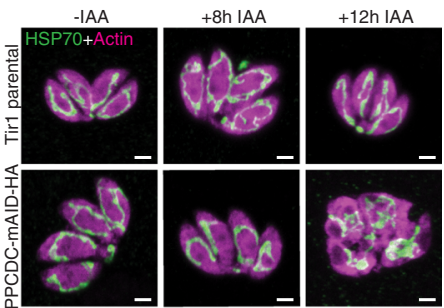

I

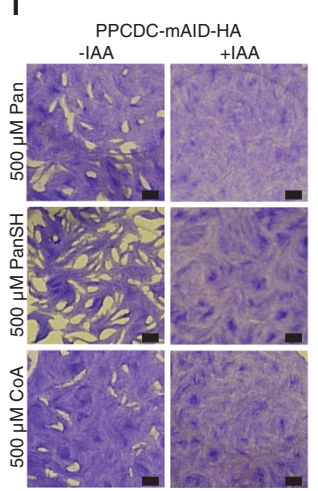

j

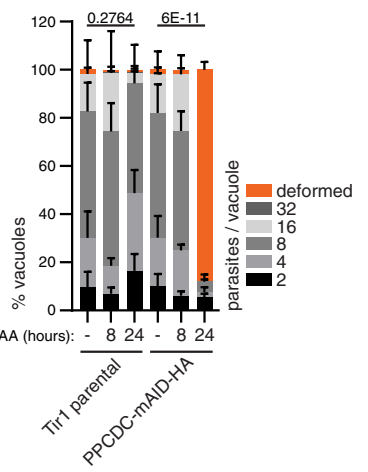

k

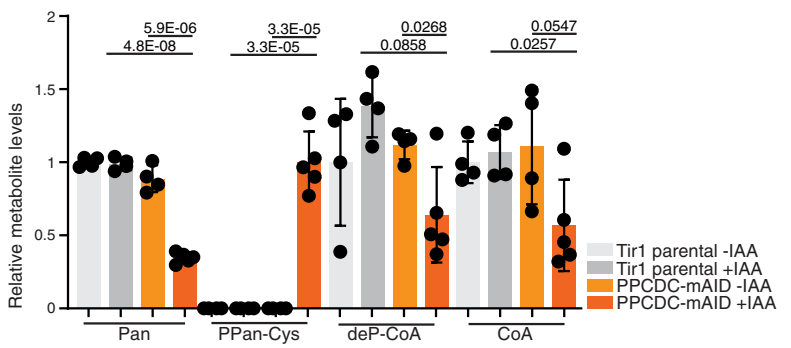

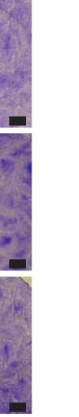

m

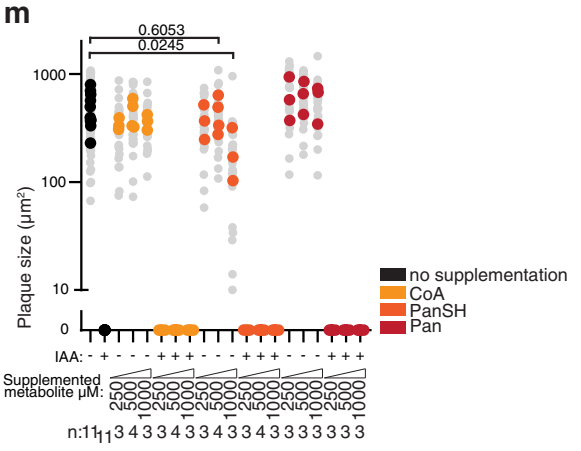

observed cell deformation, PPCDC was found to be essential for the lytic cycle of $T$. gondii, as PPCDC-mAID-HA parasites failed to form plaques in a HFF monolayer over 7 days in the presence of IAA (Fig. 3f, g). These findings indicate that salvage of PanSH or PPanSH does not contribute to CoA synthesis in T. gondii under standard culture conditions. As observed for PanK1, cell morphology, as well as the apicoplast and mitochondrion stained with anti-CPN60 and anti-heat shock protein HSP70, respectively, appeared intact and normal after $8 \mathrm{~h}$ of IAA-treatment, but the onset of deformation began at $12 \mathrm{~h}$ of IAA-treatment (Fig. 3h, i). Additionally, intracellular growth assays following $24 \mathrm{~h}$ of growth in absence of IAA or following 8 - or 24 -h of IAA- treatment of PPCDC-mAID-HA parasites were performed. No deformation or growth defects were observed after $8 \mathrm{~h}$ of IAA treatment, while PPCDC-mAID-HA parasites were largely deformed after $24 \mathrm{~h}$ of IAA treatment (Fig. 3j).

To demonstrate that PPCDC catalyzes the expected decarboxylation of 4'-phospho-N-pantothenoylcysteine (PPanCys) participating in $\mathrm{CoA}$ synthesis, targeted metabolomic analyses were performed by LC-MS on parasite extracts collected $8 \mathrm{~h}$ after IAAtreatment. Consistent with the expected function of PPCDC, its substrate PPan-Cys accumulated but was not detected in control strains (Fig. 3k). Remarkably, Pan levels were significantly decreased, indicating that accumulating PPan-Cys, or the 
Fig. 3 T. gondii cannot rely on the salvage of CoA or intermediates of the CoA synthesis pathway. a Scheme of the CoA biosynthesis pathway, highlighting putative salvage pathways of pantetheine $(\mathrm{PanSH})$ and phosphopantetheine (PPanSH) and the T. gondii phosphopantothenoylcysteine decarboxylase (PPCDC). See Fig. 1 for abbreviations. b Western blot of endogenously tagged RH PPDCD-Ty. Antibodies anti Ty, anti actin as loading control $(n=4)$. c Immunofluorescence assay (IFA) of RH PPCDC-Ty parasites. Antibodies anti Ty, anti GAP45 as marker of parasite pellicle $(n=3)$. d IFA of PPCDC-mAID-HA. Antibodies anti HA, and anti actin marking the parasite cytoplasm $(n=4)$. e IFA of $24 \mathrm{~h}$ IAA treated PPCDC-mAID-HA. Antibodies anti GAP45 for parasite pellicle, DAPI for nuclear staining $(n=3)$. $\mathbf{f}$ Plaque assay of PPCDC-mAID-HA parasites in presence of IAA ( $n=4)$. $\mathbf{g}$ Quantification of plaque size. Black dots present the average of each independent experiment. $(n=3,2$-sided $t$-test). $\mathbf{h}$, $\mathbf{i}$ IFAs of apicoplasts and mitochondria, respectively, at the indicated timepoints of IAA addition in PPCDC-mAID-HA. Antibodies anti actin (cytoplasm), anti CPN60 (apicoplast), anti HSP70 (mitochondrion) $(n=3)$. $\mathbf{j}$ Intracellular growth assay of PPCDC-mAID-HA. Deformed parasites scored as indicated in Fig. $2 \mathrm{~h}(n=3-6, \mathrm{mean}$ and SD, 2 tailed $t$-test on the "deformed" category). $\mathbf{k}$ LC-MS analysis of metabolite abundances in PPCDC depleted parasites ( $8 \mathrm{~h}+\mathrm{IAA}, n=4-5, \mathrm{mean}$ and SD, 2-sided Student's $t$ test). Metabolite levels normalized to internal standard $\left({ }^{13} \mathrm{C}_{6} /{ }^{15} \mathrm{~N}\right.$-isoleucine) and quantified relative to Tir1 parental $-I A A$. I Plaque assay of PPCDC-mAID-HA parasites in presence of IAA, with the supplementation of $500 \mu \mathrm{M}$ Pan, pantetheine $(\mathrm{PanSH})$, or CoA ( $n=3)$. m Quantification of plaque size. Colored dots represent the average of each independent experiment ( $n$ indicated in the panel, 2-sided $t$-test). The Tir1 parental controls of Fig. 3d-k are shared with Figs. 2c-f, i-I. n number of independent biological replicates. Source data are provided as a Source Data file. White scale bar $2 \mu \mathrm{m}$, black scale bar $1 \mathrm{~mm}$.

upstream intermediate PPan (not measured) may provide a feedback signal to reduce Pan acquisition. Crucially, the metabolites downstream of PPCDC, deP-CoA and CoA were significantly reduced upon PPCDC-downregulation compared to the control strains. Of note, the values for the Tirl parental control correspond to those shown in Fig. 2m, as these samples were analyzed in a single batch. Lastly, we investigated whether exogenously supplemented CoA, or its precursors, could facilitate their salvage and overcome the essentiality of PPCDC. However, neither supplementation of Pan, nor PanSH or CoA (each provided at $250 \mu \mathrm{M}, 500 \mu \mathrm{M}$ and $1 \mathrm{mM}$ ) were able to rescue the parasite's inability to form lysis plaques upon downregulation of PPCDC (Fig. 31, m, Supplementary Fig. 1d). At the highest concentration tested here, $1 \mathrm{mM}$, PanSH supplementation caused the formation of smaller plaques (Fig. $3 \mathrm{~m}$, Supplementary Fig. 1d), pointing towards toxicity of this intermediate at high concentrations. Thus, neither PanSH salvage pathway, nor direct salvage of CoA or $\mathrm{PPanSH}$, following CoA hydrolysis, contribute (sufficiently) to the CoA pool of T. gondii even when provided in excess. Whether this is due to inefficient salvage by the host, the parasite, or less promiscuous PanKs compared to $P$. yoelii and $P$. berghei, remains unclear.

In conclusion, PPCDC is an essential enzyme in the CoA synthesis pathway of $T$. gondii that generates $\mathrm{PPanSH}$, and its function cannot be bypassed by the salvage of downstream metabolites. Lack of PPCDC leads to accumulation of its substrate and a drop in CoA levels, which causes severe morphological defects and eventually parasite death.

The final step of CoA biosynthesis is essential in T. gondii and relies on a membrane-bound cytoplasmic DPCK. The gene coding for the final CoA synthesis enzyme, DPCK was recently identified upstream of the alanine dehydrogenase gene in the $T$. gondii genome (TGME49_315260) ${ }^{9}$. DPCK catalyzes the phosphorylation of dephospho-coenzymeA (deP-CoA) (Fig. 4a). The products of a second copy C-terminally myc-tagged DPCK under the constitutive tubulin promoter (cDPCK-myc) and the C-terminally spaghetti monster-myc ${ }^{24}$ tagged DPCK (DPCKSMmyc) at the endogenous locus migrated by western blot close to the expected sizes of $37 \mathrm{kDa}$ and $72 \mathrm{kDa}$, respectively (Fig. $4 \mathrm{~b}$ ). In accordance with the presence of two predicted transmembrane domains, cDPCK-myc and DPCK-SMmyc behave as integral membrane proteins based on fractionation experiments (Fig. 4c, d). cDPCK-myc and DPCK-SMmyc were detected in the pellet fraction of parasite lysates upon sodium carbonate $\left(\mathrm{Na}_{2} \mathrm{CO}_{3}\right)$ extraction, but in the soluble fraction upon extraction with the detergents Triton X-100 (Tx-100) or sodium dodecyl sulfate (SDS). In contrast to P. falciparum DPCK, which localizes to the apicoplast ${ }^{25}$, TgDPCK revealed a dotty cytoplasmic localization by IFA (Fig. 4e), clearly distinct from the apicoplast (see Fig. 4j for comparison) and in concordance with the localization of all other tagged genes in the CoA synthesis pathway. Depletion of DPCK was efficiently achieved using the tet-repressive promoter system, in an RH ku80-ko hxgprt-ko (RH parental) in presence of anhydrotetracycline (ATc) as demonstrated by western blot (Fig. 4f, Supplementary Fig. 1e) ${ }^{26}$. Important for the interpretation of these results, stage specificity is not an issue with these minimal tet promoters ${ }^{26,27}$. Protein downregulation, using the promoter repression system, takes effect considerably slower than the rapid IAA-induced protein degradation system, with residual protein detected after $24 \mathrm{~h}$ (Fig. $4 \mathrm{f}$ ). Depletion of DPCK over $48 \mathrm{~h}$, resulted in dramatic loss of parasite morphology (Fig. 4g). Consistent with this, prolonged ATc-treatment of DPCK-iKD parasites prevented the formation of lysis plaques in a HFF monolayer over 7 days of growth (Fig. 4h), quantified in Fig. 4i. Critically, this defect in the lytic cycle was rescued by the expression of a second copy DPCK-myc (DPCK-iKD::cDPCK-myc, Fig. 4h, i). Parasite morphology and growth were assessed following ATctreatment for varying durations including $0,32,48$ and $72 \mathrm{~h}$ of ATc (Fig. 4j-1). While aberrant morphology was observed after $48 \mathrm{~h}$ of ATc-treatment for the total cell, the apicoplast and the mitochondrion appeared intact after $32 \mathrm{~h}$ of treatment. However, a slight defect in growth and a small percentage of deformed parasites were observed after $32 \mathrm{~h} \mathrm{ATc}$, which aggravated over longer durations of ATc-treatment (48 and $72 \mathrm{~h}$ ) (Fig. 4l). As observed for the plaque assay, defects in intracellular growth and morphology were rescued by expression of a second copy DPCKmyc (Fig. 4l).

To assess DPCK activity, metabolite extracts of parental and DPCK depleted parasites strains were analyzed by LC-MS on extracts collected after 32 and $72 \mathrm{~h}$ of ATc-treatment. Upon $32 \mathrm{~h}$ ATc-induced DPCK downregulation, Pan levels were unaltered, while deP-CoA levels increased significantly (3 fold), consistent with a block of the expected enzymatic function, the phosphorylation of deP-CoA (Fig. $4 \mathrm{~m}$ ). CoA levels were unaltered at this relatively early time-point of DPCK-downregulation, whereas at the time point of $72 \mathrm{~h}$ ATc-treatment, DPCK depleted parasites presented significantly higher Pan and 15-fold increased levels of the substrate deP-CoA but markedly reduced levels of CoA $(\sim 10$ fold) and the active metabolite acetyl-CoA (2-3-fold) (Fig. 4n). While DPCK-iKD parasites are expected to be unfit and partially deformed at this stage based on our phenotyping described above, these experiments validate the enzymatic function of DPCK. The high levels of accumulating Pan and deP-CoA measured in these parasite extracts support that these parasites still have intact membranes and at least a partially functional metabolism. 

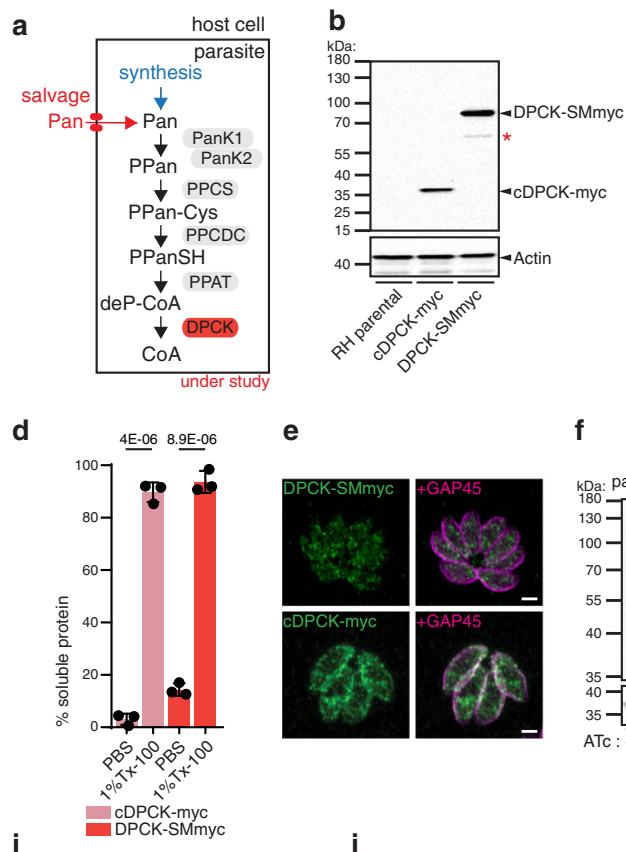

$\mathbf{f}$
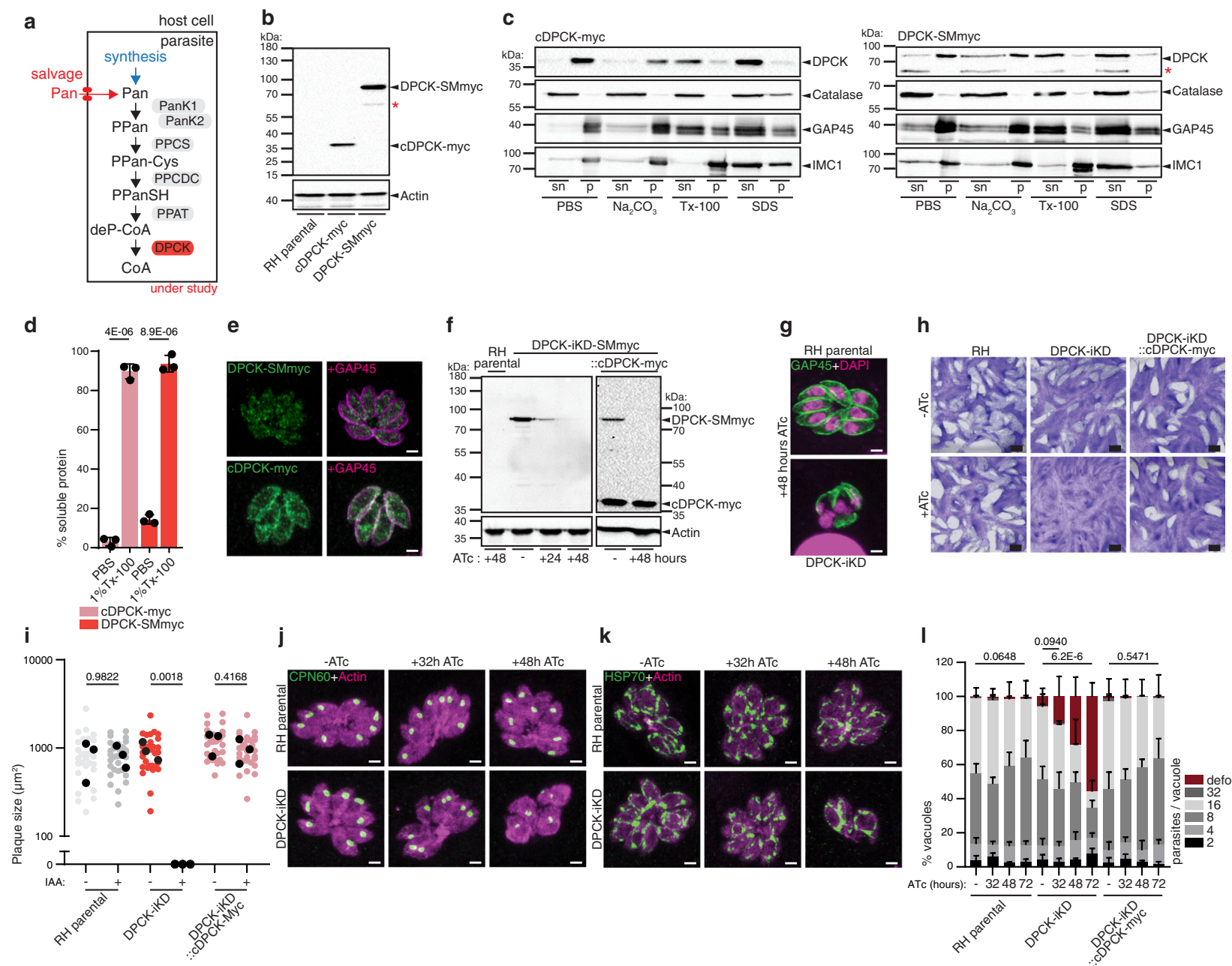

DPCK-iKD

m
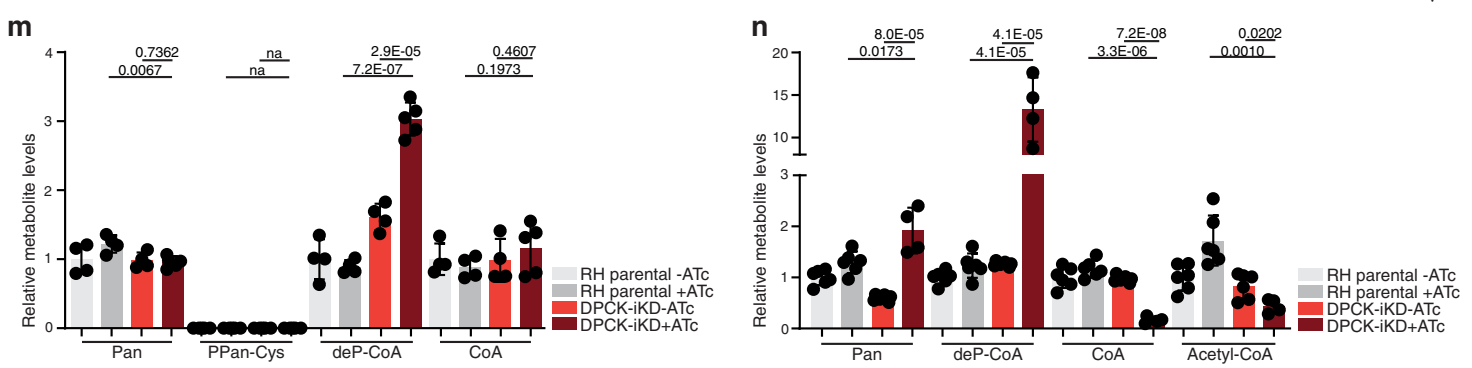

Fig. 4 The final step of CoA biosynthesis is essential in T. gondii and relies on a membrane-bound cytoplasmic DPCK. a Scheme of CoA biosynthesis pathway, highlighting the final CoA synthesis enzyme dephospho-CoA kinase (DPCK). See Fig. 1 for abbreviations. b Western blot of cDPCK-myc (expected MW $37 \mathrm{kDa}, n=3$ ) and spaghetti-monster (SM)myc tagged DPCK (expected MW $72 \mathrm{kDa}, n=3$ ). Antibodies anti myc and anti actin as loading control. c Western blot of solubility assay of cDPCK-myc $(n=3)$ and DPCK-SMmyc $(n=3)$. Antibodies anti myc/ anti-catalase, anti GAP45 and anti IMC1 as markers for PBS-, Triton- and SDS-soluble fraction, respectively. sn supernatant, p pellet. Solubility quantified in $\mathbf{d}(n=3$, mean and SD). e IFA of cDPCKmyc $(n=3)$ and DPCK-SMmyc tagged strains $(n=3)$. Antibodies: anti myc, anti GAP45 as marker of parasite pellicle. f Western blot of DPCK-iKD-SMmyc and cDPCK-myc protein levels upon anhydrotetracycline (ATc) addition. Antibodies anti myc, anti actin as loading control $(n=3)$. $\mathbf{g}$ IFA of $48 \mathrm{~h}$ ATc treated DPCK-iKD. Antibodies: anti GAP45 (parasite pellicle), DAPI (nucleus) $(n=3)$. h Plaque assay of DPCK-iKD and DPCK-iKD:::cDCPK-myc $(n=3)$. $\mathbf{i}$ Quantification of plaque size. Black dots present the average of each independent experiment. $(n=3,2$-sided $t$-test). $\mathbf{j}$, $\mathbf{k}$ IFAs of apicoplasts and mitochondria in DPCK-iKD, at the indicated timepoints of ATc addition. Antibodies: anti actin (cytoplasm), anti-CPN60 (apicoplast), anti-HSP70 (mitochondrion) $(n=3)$. I Intracellular growth assay of RH and DPCK-iKD parasites over $24 \mathrm{~h}$. Deformed parasites scored as indicated in Fig. $1 \mathrm{~h}$ ( $n=3-6$, mean and SD, 2 tailed $t$-test on "deformed" category). $\mathbf{m}, \mathbf{n}$ LC-MS analysis of metabolite abundances in DPCK depleted parasites ( $32 \mathrm{~h}$ ATc, $\mathrm{m} ; 72 \mathrm{~h}$ ATc, n). $n=4-5$ for $\mathbf{m}$ and 5-6 for $\mathbf{n}$, mean and SD, 2-sided Student's $t$ test). Metabolite levels normalized to internal standard $\left({ }^{13} \mathrm{C}_{6} /{ }^{15} \mathrm{~N}\right.$-isoleucine for $\mathrm{n}$ and ${ }^{13} \mathrm{C}_{3} /{ }^{15} \mathrm{~N}$-pantothenate for $\mathrm{m}$ ) and quantified relative to $\mathrm{RH}-\mathrm{ATc}$. $n$ number of independent biological replicates. Source data are provided as a Source Data file. White scale bar $2 \mu \mathrm{m}$, black scale bar $1 \mathrm{~mm}$. Red asterisks indicate degradation products of the tagged protein. 
Taken together, the combination of reverse genetics and metabolomic analyses firmly confirms the enzymatic function of four (PanK1 +2, PPCDC, DPCK) of the six T. gondii CoA biosynthesis enzymes, catalyzing five steps. Critically, a block at any of the three investigated steps in CoA synthesis pathway results in severe depletion of essential metabolites, such as CoA and acetylCoA, ultimately causing parasite death. Several studies have highlighted the druggability of the pathway in Apicomplexa, due to the inhibition of acetyl-CoA generating enzymes ${ }^{28-30}$. Our results uncover that specific inhibition of $T$. gondii CoA synthesis enzymes could be an effective treatment against toxoplasmosis and cannot be overcome by salvage of CoA or other intermediates.

The Pan synthesis pathway is dispensable due to Pan uptake in tachyzoites. While CoA can be produced by all organisms, humans and animals are unable to synthesize its precursor Pan, which is synthesized by plants, fungi and most bacteria ${ }^{31}$. Some protists (mainly Alveolata and Stramenopiles) encode in their genome the required enzymatic pathway. T. gondii encodes the three proteins required for de novo synthesis of Pan (Fig. 5a): i) the branched chain amino acid transaminase (BCAT), previously localized to the mitochondrion and shown to be active but dispensable in tachyzoites ${ }^{32}$; ii) the bifunctional enzyme ketopantoate hydroxymethyltransferase - ketopantoate reductase, (KPHMT-KPR) and iii) the pantoate $\beta$-alanine ligase (PBAL, also known as Pan synthetase, encoded by panC in E.coli) $)^{9,10,33,34}$. In contrast to model organisms, alveolates encode the pathway with various gene duplications and rearrangements, like for the bifunctional KPHMT-KPR in T. gondii (Fig. 1a, b, Supplementary Data 1).

To investigate the localization of Pan synthesis in T. gondii, KPHMT-KPR (TGME49_257050) and PBAL (TGME49_265870) were C-terminally epitope- tagged at the endogenous locus with $\mathrm{SMmyc}^{24}$ and $3 \mathrm{xTy}$, respectively. Both tagged proteins migrated on western blots close to the predicted sizes of $144 \mathrm{kDa}$ and $93 \mathrm{kDa}$, respectively (Fig. 5b). KPHMT-KPR-SMmyc co-localized with the mitochondrial marker HSP70, while PBAL-Ty localized to the nucleus (Fig. 5c). These findings point towards pantoate, as has been described for ketopantoate in plant model organisms ${ }^{35}$, being synthesized in the mitochondrion and transported into the cytosol/nucleus, where it is converted into Pan by PBAL. Furthermore, these observations, together with a previous study of $\mathrm{BCAT}^{32}$, reveal that all Pan synthesis genes are expressed in tachyzoites. To assess the importance of Pan synthesis for $T$. gondii fitness, KPHMT-KPR and PBAL genes were deleted by Cas9-mediated double homologous recombination (Supplementary Fig. 2a). Consistent with a published genome-wide fitness score suggesting dispensability ${ }^{15}$, parasites lacking Pan synthesis enzymes exhibited no defect in the lytic cycle compared to the parental RH strain (Fig. 5 d, e). These results stand in contrast to a previous pharmacological study ${ }^{33}$, where the efficacy of $M y c o-$ bacterium tuberculosis Pan synthetase inhibitors was tested against $T$. gondii tachyzoites in vitro. The authors reported growth inhibition in the nanomolar range for the compounds SW413 and SW404, which was partially rescued by supplementation with high levels of exogeneous $\mathrm{Pan}^{33}$. However, efficient growth reduction through inhibition of $T$. gondii $\mathrm{PBAL}$ is incompatible with the dispensability of the $P B A L$ gene reported here and supported by the genome-wide fitness screen ${ }^{15}$. In consequence, the reported efficacy of SW413 and SW404 against T. gondii is possibly owed to off-target effects. Competitive inhibition of the PanKs rather than PBAL by the compounds, could potentially explain the growth inhibition and the partial rescue through excess Pan.
Of relevance, the dispensability of the Pan synthesis enzymes implies that $T$. gondii tachyzoites are capable of salvaging Pan. Pan may be taken up from the host and also directly from the culture medium during extracellular periods where it is present at 8.3 or $0.5 \mu \mathrm{M}$ (Dulbecco's Modified Eagle Medium, DMEM; Roswell Park Memorial Institute, RPMI, respectively). To examine whether tachyzoites can take up exogeneous Pan, stable isotope labeling followed by LC-MS was performed. Isolated extracellular parasites were incubated for $15 \mathrm{~min}$ in medium containing $40 \mu \mathrm{M}{ }^{13} \mathrm{C}_{3} /{ }^{15} \mathrm{~N}$-Pan and for reference $1 \mathrm{mM}{ }^{13} \mathrm{C}_{6} /$ ${ }^{15} \mathrm{~N}$-isoleucine (ILE), before quenching the metabolism and washing cells rigorously. Both metabolites were readily taken up within this short time frame leading to significant increase in the abundance of the M7 and M4 isotopologues of ILE and Pan, respectively, as measured by LC-MS (Fig. 5f).

To further scrutinize whether T. gondii takes up exogeneous Pan, stable isotope labeled Pan $\left({ }^{13} \mathrm{C}_{3} /{ }^{15} \mathrm{~N}-\mathrm{Pan}, 100 \mu \mathrm{M}\right)$ was added to the culture media for $40 \mathrm{~h}$ to infected HFFs. This led to pronounced labeling $(80 \%)$ of the intracellular Pan pool, as measured by gas chromatography-mass spectrometry (GC-MS) analysis of parasite extracts, indicating efficient uptake of the vitamin (Fig. 5g, Supplementary Fig. 2b). These findings demonstrate that T. gondii tachyzoites, as P. falciparum ${ }^{36}$ and as expected for other apicomplexans, is capable of salvaging Pan. While essentiality of Pan uptake in Plasmodium spp. was proposed several decades ago ${ }^{37}$ and a putative Pan transporter was proposed in $P$. falciparum $^{38}$, subsequent studies provided evidence that this putative Pan transporter localizes to secretory organelles and is implicated in exocytosis both in P. berghei and in T. gondii ${ }^{39,40}$. Specifically, T. gondii depleted in the closest homolog (Transporter Facilitator Protein 1, TFP1) of the putative P. falciparum Pan transporter (PfPAT) displayed no intracellular growth defect, which is highly atypical for proteins involved in metabolism, but rather presented impaired host cell attachment, invasion and egress ${ }^{40}$. Thus, the apicomplexan Pan transporter remains to be identified, as a putative target for intervention against these important group of human pathogens. To investigate the importance of Pan salvage in T. gondii, tachyzoites were grown in custom-made culture medium lacking Pan and supplemented with dialyzed fetal bovine serum (dFBS). The absence of Pan in the culture medium including 5\% dFBS was confirmed by GC-MS analysis (Supplementary Fig. 3). Unexpectedly, neither wildtype (RH parental) nor pbal-ko parasites cultivated in medium depleted of Pan displayed reduced fitness or altered growth rates, as shown by plaque assay and intracellular growth assay (Fig. 5h-j). To investigate how $T$. gondii parasites cope under these Pan-limiting conditions, GCand LC-MS analyses were employed to directly measure the intracellular levels of Pan and its downstream metabolites, dePCoA, CoA and acetyl-CoA. Parasites cultured in Pan-depleted medium for two or three passages in pre-depleted host cells indeed presented a 2-3-fold decrease but were not completely devoid of Pan (Fig. 5k, l). From the second to the third consecutive passage of parasites in host cells pre-depleted of Pan, intracellular Pan levels did not decrease further (Fig. 5k). Strikingly, while CoA and its precursors were significantly decreased in parasite extracts, acetyl-CoA levels were unchanged or even increased upon Pan-depletion, indicating that even low levels of Pan are sufficient to synthesize ample amounts of this critical metabolite (Fig. 5k).

The parasite's ability to grow in medium deficient of Pan was previously reported, with the authors concluding that $T$. gondii relies on Pan synthesis ${ }^{28}$. However, depletion of PBAL had no impact on intracellular Pan-levels in parasites cultivated either in regular or Pan-depleted culture media (Fig. 5l), as measured by 
a

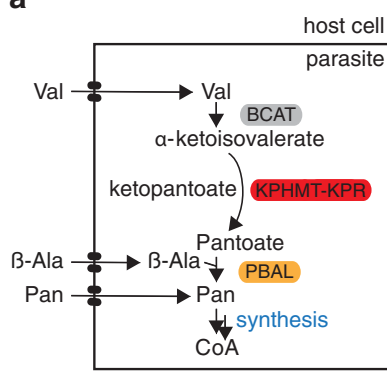

b

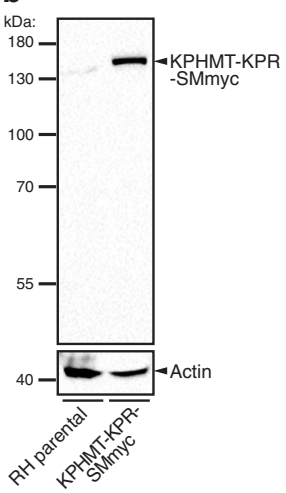

c

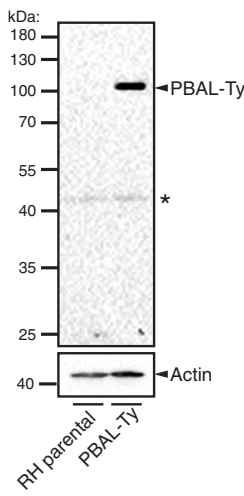

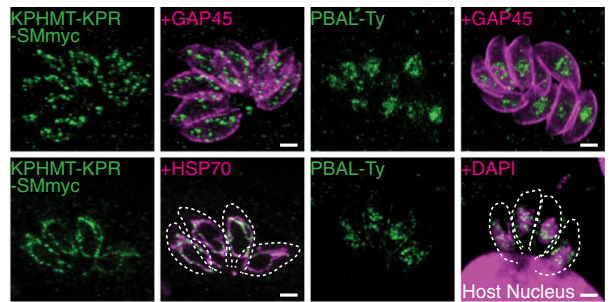

d

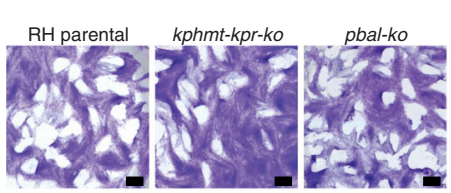

e

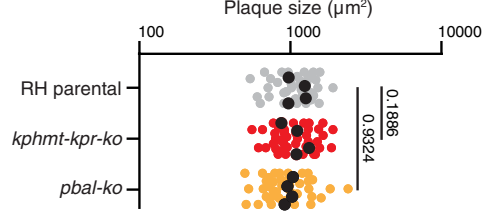

f

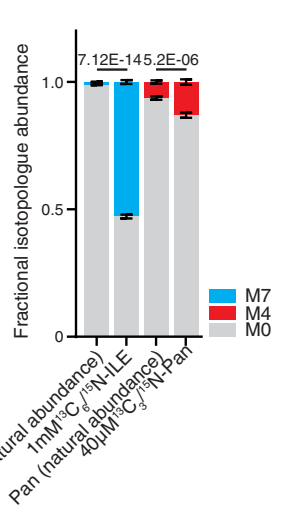

g

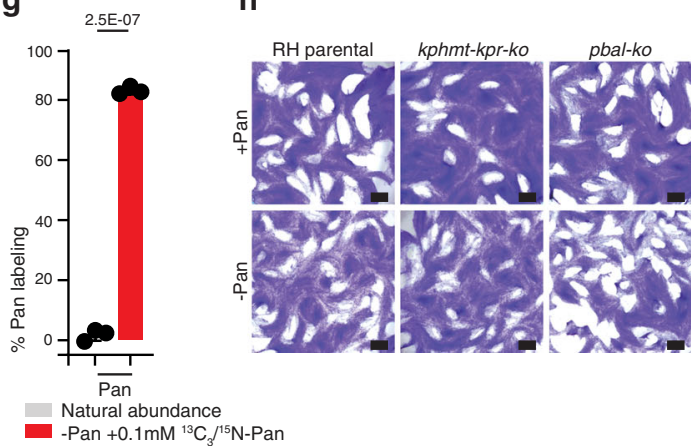

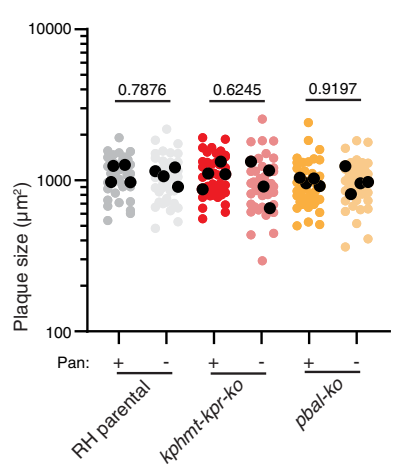

j

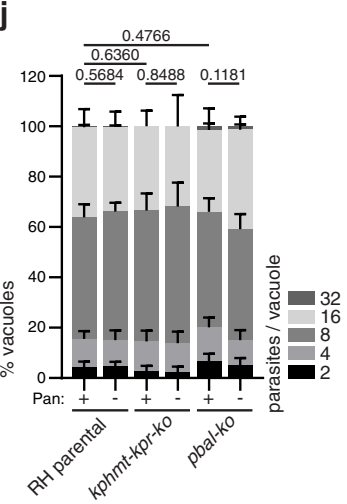

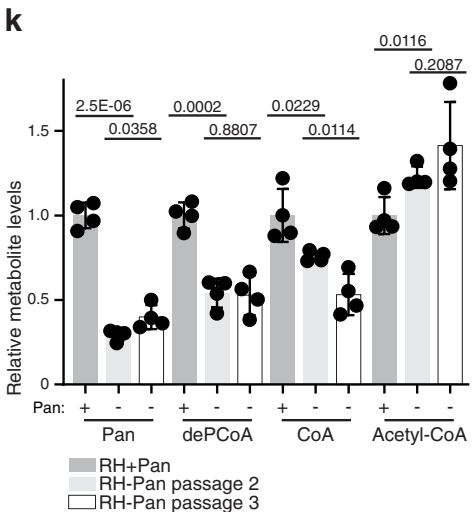

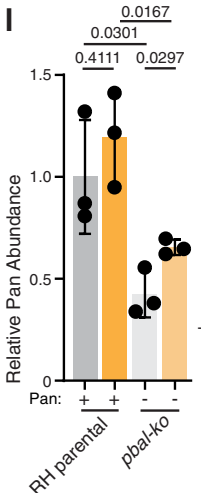

+ Pan $\mid \begin{gathered}\text { RH parental } \\ \text { pbal-ko }\end{gathered}$ \begin{tabular}{l|l} 
pbal-ko \\
RH parental \\
pbal-ko
\end{tabular}

Fig. 5 The Pan synthesis pathway is dispensable due to Pan uptake in tachyzoites. a Scheme of the Pan biosynthesis pathway, highlighting the ketopantoate hydroxymethyltransferase - ketopantoate reductase (KPHMT-KPR) and the pantoate beta alanine ligase (PBAL). See Fig. 1 for abbreviations. b Western blots of endogenous C-terminally spaghetti-monster (SMmyc) tagged KPHMT-KPR (MW $144 \mathrm{KDa}, n=3$ ) and endogenous C-terminally Ty tagged PBAL (MW $93 \mathrm{KDa}, n=3$ ), respectively. Antibodies anti-myc and anti-Ty, anti actin as loading controls. c Immunofluorescence assay of KPHMTKPR-SMmyc $(n=3)$ and PBAL-Ty $(n=5)$. Antibodies anti-myc and anti-Ty, anti GAP45 (pellicle), anti HSP70 (mitochondrion), DAPI (nucleus). d Plaque assay of RH parental, kphmt-kpr-ko $(n=4)$ and pbal-ko $(n=6)$. e Quantification of plaque size. Black dots present the average of each independent experiment. ( $n=4,2$-sided $t$-test). $\mathbf{f L C}$-MS analysis of Pan (MO, M4) and isoleucine (MO, M7) isotopologues, following 15 min incubation of isolated RH parasites in medium $+40 \mu \mathrm{M}^{13} \mathrm{C}_{3} /{ }^{15} \mathrm{~N}$-Pan and $1 \mathrm{mM}{ }^{13} \mathrm{C}_{6} /{ }^{15} \mathrm{~N}$-isoleucine (ILE) ( $n=3-6$, mean and SD, 2-sided Student's $t$ test). $\mathbf{g}$ GC-MS analysis of RH parental derived Pan upon culture in medium containing ${ }^{13} \mathrm{C}_{3} /{ }^{15} \mathrm{~N}$-Pan $(100 \mu \mathrm{M})$ for $40 \mathrm{~h}(n=4$, mean and SD, 2-sided Student's $t$ test). h Plaque assay of mutant parasites in \pm Pan culture medium $(n=4)$. i Quantification of plaque size. Black dots present the average of each independent experiment. ( $n=4$, 2 -sided $t$-test). $\mathbf{j}$ Intracellular growth assay of mutant parasites in \pm Pan culture medium ( $n=3-6$, mean and SD, 2 tailed $t$-test on the " 16 parasites/vacuole" category). k LC-MS measurement of intracellular Pan, dephospho-CoA (deP-CoA), coenzyme A (CoA) and acetyl-CoA ( AcCoA) in RH parental parasites grown for 2 and 3 passages (P2, P3) in \pm Pan culture media. Metabolite levels normalized to internal standard $\left({ }^{13} \mathrm{C}_{3} /{ }^{15} \mathrm{~N}\right.$-Pan) and quantified relative to $\mathrm{RH}+\operatorname{Pan}(n=4$, mean and SD, 2-sided Student's $t$ test). I GC-MS measurement of Pan levels in RH parental and pbal-ko parasites grown in \pm Pan culture medium. Metabolite levels normalized to signal intensity of the total ion chromatogram and quantified relative to RH + Pan. ( $n=4$, mean and SD, 2-sided Student's $t$ test). $n$ number of independent biological replicates. Source data are provided as a Source Data file. White scale bar $2 \mu \mathrm{m}$, black scale bar $1 \mathrm{~mm}$. Asterisks mark unspecific bands. 
GC-MS, indicating that salvage of residual Pan rather than synthesis is responsible for the remaining Pan detected in parasites following depletion. To determine if tachyzoites are able to modulate the expression of host Pan recycling enzymes such as pantetheinases and CoA pyrophosphatases under Pan depletion conditions ${ }^{41}$, RNA-seq analyses were performed on parasites sequentially passaged in Pan-depleted HFFs. A total of 621 transcripts were significantly changed (409 genes up- and 212 downregulated with $p$ value $<0.01$ ) in the parasite transcriptome (Supplementary Data 2), but none displayed changes greater or lesser than 4 -fold ( $>2 \log _{2}$ fold). In the transcriptome of parasiteinfected HFFs, infection with Pan-deprived parasites compared to parasites grown in rich-media, significantly altered the levels of 1691 transcripts (871 genes up- and 820 downregulated with $p<0.01$, from Supplementary Data 2), 92 of which differentially regulated more than 4 -fold (Supplementary Data 2). The genes participated in diverse cellular processes (GO terms in Supplementary Data 2), but none that could indicate an increased salvage mechanism or any alteration to the Pan/CoA metabolism within the host.

Taken together, these results reveal that a complete depletion in intracellular Pan cannot be readily achieved when cultivating T. gondii tachyzoites in HFFs. As long as the host cell viability is not compromised, the parasite is able to salvage sufficient Pan from its host. Due to this efficient salvage, Pan synthesis is dispensable in cultured T. gondii tachyzoites.

T. gondii tachyzoites salvage Pan synthesis intermediates and uses these for Pan synthesis. To interrogate further whether the $T$. gondii Pan synthesis pathway can contribute to the parasite's metabolite pool under Pan-limiting conditions, Pan synthesis was promoted by supplementing the culture medium with excess of PBAL substrates in the absence of exogenous Pan. Valine (Val), an essential amino acid for $T$. gondii and the substrate of BCAT (Fig. 6a), is abundantly present in culture medium $(0.8 \mathrm{mM}$ in DMEM) and is readily detected in parasite extracts ${ }^{42}$ (Supplementary Fig. 4a). In contrast, the non-proteinogenic amino acid beta-alanine $(\beta-\mathrm{Ala})$ is absent in standard culture medium, including DMEM, but is also detected at low levels in extracts of parasites cultured under standard conditions using GC-MS and LCMS (Supplementary Fig. 4a, b). It is unclear whether T. gondii is capable of synthesizing $\beta$-Ala since at least 6 different $\beta$-Ala synthesis pathways have been described in model organisms, some of which involve potentially promiscuous transaminases and aldehyde dehydrogenases ${ }^{43}$. Since mammalian host cells are capable of producing $\beta$-Ala ${ }^{44}, T$. gondii might rely on its salvage. The parasite's ability to take up exogeneous $\beta$-Ala was clearly demonstrated in experiments described below. In bacteria, $\beta$-Ala has been shown to be a limiting factor for Pan synthesis, with elevated $\beta$-Ala levels leading to increased Pan synthesis ${ }^{45}$. However, supplementation of $\mathrm{Val}$ and $\beta$-Ala at $3 \mathrm{mM}$ and $2 \mathrm{mM}$, respectively, to the culture medium failed to restore or increase Pan levels in parasites depleted of exogeneous Pan, as measured by GC-MS (Fig. 6b). These results suggest that these precursors cannot be the only limiting factors for Pan synthesis and provide further evidence that residual intracellular Pan in depletion experiments is derived from salvage rather than its de novo synthesis. To investigate more specifically whether Pan synthesis from Val and $\beta$-Ala (Fig. 6a) is functional and active in T. gondii by means of a more sensitive approach, stable isotope labeling combined with LC-MS was employed. Parasites were incubated in medium containing stable isotope labeled ${ }^{13} \mathrm{C}_{3} /{ }^{15} \mathrm{~N}-\beta$ Ala $(3 \mathrm{mM})$ or ${ }^{13} \mathrm{C}_{3} /{ }^{15} \mathrm{~N}$-Pan $(100 \mu \mathrm{M})$ for $48 \mathrm{~h}$. During active Pan synthesis, stable isotopes from ${ }^{13} \mathrm{C}_{3} /{ }^{15} \mathrm{~N}-\beta$-Ala are expected to be incorporated into de novo synthesized Pan and further into CoA (see cartoon in Fig. 6c). However, intracellular Pan and its derived metabolites, CoA and acetyl-CoA, remained unlabeled following growth of parasites in medium containing ${ }^{13} \mathrm{C}_{3} /{ }^{15} \mathrm{~N}-\beta$-Ala (Fig. $6 \mathrm{~d}$ ). In contrast, incubation of the parasites in medium containing ${ }^{13} \mathrm{C}_{3} /$ ${ }^{15} \mathrm{~N}$-Pan resulted in labeling of the intracellular Pan pool, and the labeled Pan was readily incorporated into CoA and acetyl-CoA (Fig. 6d), confirming efficient Pan salvage, as described above (Fig. 5f, g), and revealing that salvaged Pan but not $\beta$-Ala is readily used for CoA synthesis. Importantly the lack of Pan synthesis from $\beta$-Ala is not due to insufficient uptake of ${ }^{13} \mathrm{C}_{3} /{ }^{15} \mathrm{~N}-\beta$-Ala since stable isotope labeled $\beta$-Ala was abundantly detected in parasites cultured in its presence (Supplementary Fig. 5). It presumably passes the plasma membrane through an as of yet unidentified specific or promiscuous amino acid transporter.

In consequence, de novo Pan synthesis is inactive in tachyzoites, raising the question whether these enzymes are functional. Although the catalytic residues are clearly conserved in PBAL (Supplementary Data 3), the protein is divergent and considerably larger compared to the enzymes described in other organisms $(88 \mathrm{kDa}$, vs $31 \mathrm{kDa}$ for E. coli PBAL (also called pantothenate synthetase) and $35 \mathrm{kDa}$ for Saccharomyces cerevisiae $\mathrm{PBAL}$ ). Recombinant expression of full length PBAL in either bacteria or Sf9 insect cells did not yield any folded enzyme, and a construct composed of the bacterial maltose binding protein (MBP) fused to the predicted catalytic domain of PBAL (PBALcd, residues 109-492) could be purified to high purity from E. coli (Supplementary Fig. 6) but did not show reliable enzymatic activity when incubated with its substrates $\beta$-Ala and pantoate in vitro, in contrast to the bacterial PanC enzyme. Of relevance, this PBAL construct likely forms large soluble oligomers as it elutes in the void volume when analyzed by size exclusion chromatography. It is thus conceivable that proper folding of active recombinant PBAL requires additional partners or chaperones.

To interrogate which step(s) of Pan synthesis is/are inactive, preventing its de novo synthesis from $\mathrm{Val}$ and $\beta$-Ala, mutants of each Pan synthesis pathway enzyme were employed (bcat-ko ${ }^{32}$, $k p h m t-k p r-k o, p b a l-k o$ ). Wildtype parasites (RH parental) and the mutants were cultured in the presence or absence of various Pan precursors (all $0.8 \mathrm{mM}$ ) in medium lacking Pan, while Val was present in all conditions at $0.8 \mathrm{mM}$ (default concentration in DMEM). Pan and its precursors were identified in parasite extracts by LC-MS based on their retention time, $\mathrm{m} / \mathrm{z}$ of the parental ion and $\mathrm{MS}^{2}$ spectra of authentic standards (Supplementary Fig. 7a). The activity of each Pan synthesis enzymes was probed by supplementing the culture medium with stable isotope labeled ${ }^{13} \mathrm{C}_{3} /{ }^{15} \mathrm{~N}-\beta$-Ala $(0.8 \mathrm{mM})$ for $40 \mathrm{~h}$, but, as above, presence of Val and $\beta$-Ala was insufficient to trigger Pan synthesis, as judged by the absence of labeled Pan (Fig. 6e, Supplementary Fig. 9a). In order to bypass the initial enzyme (BCAT), aketoisovalerate was supplemented in addition to ${ }^{13} \mathrm{C}_{3} /{ }^{15} \mathrm{~N}-\beta$-Ala (both $0.8 \mathrm{mM}$ ). Interestingly, $\alpha$-ketoisovalerate was detected at very low levels in extracts of non-supplemented parasites, indicating that BCAT is active, as previously reported ${ }^{32}$ (Supplementary Fig. 7b). When supplemented at $0.8 \mathrm{mM}$, the signal intensity of intracellular a-ketoisovalerate only increased marginally (Supplementary Fig. 7c), highlighting that its acquisition by the parasite or the host is inefficient and/or salvaged $\alpha$ ketoisovalerate is rapidly metabolized. Crucially, provision of exogeneous $\alpha$-ketoisovalerate and ${ }^{13} \mathrm{C}_{3} /{ }^{15} \mathrm{~N}$ - $\beta$-Ala did not result in the formation of labeled Pan (Fig. 6e, Supplementary Fig. 8a). Taken together, these findings point towards inactivity of the second enzyme, KPHMT-KPR, or low levels of intracellular $\alpha$ ketoisovalerate are not sufficient to promote Pan synthesis. Consistent with inactivity of KPHMT-KPR, pantoate was not detected in parasites cultured in non-supplemented culture medium (Supplementary Fig. 7b). Lastly, BCAT as well as the 

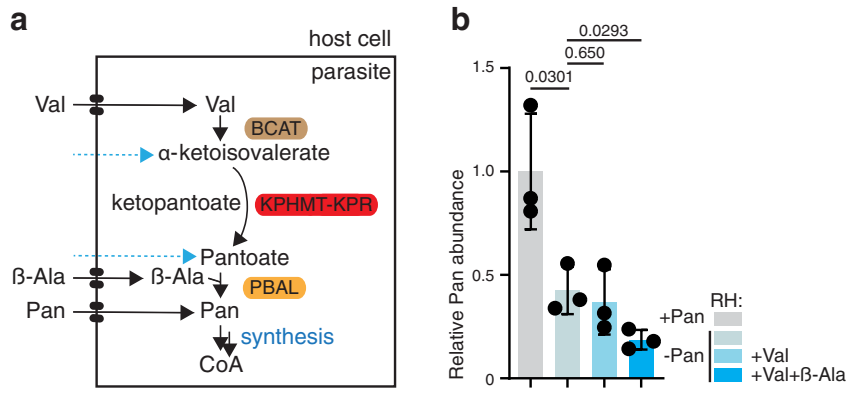

C

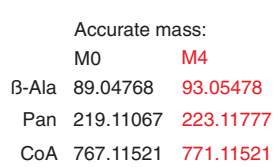<smiles>CC(C)(CO)C(O)C(=O)NCCC(=O)O</smiles><smiles>Nc1ncnc2c1ncn2[C@@H]1OC(COP(=O)(O)O)[C@@H](OP(=O)(O)O)[C@H]1O</smiles>

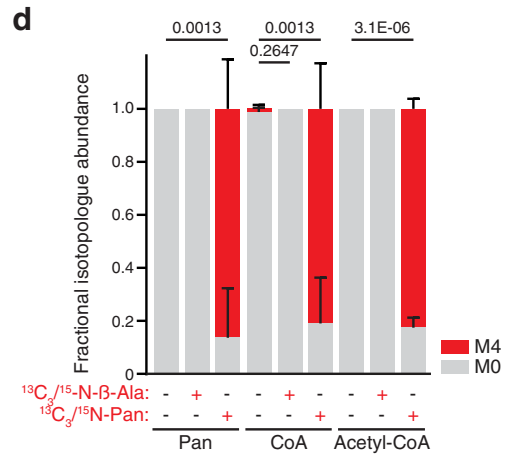

e
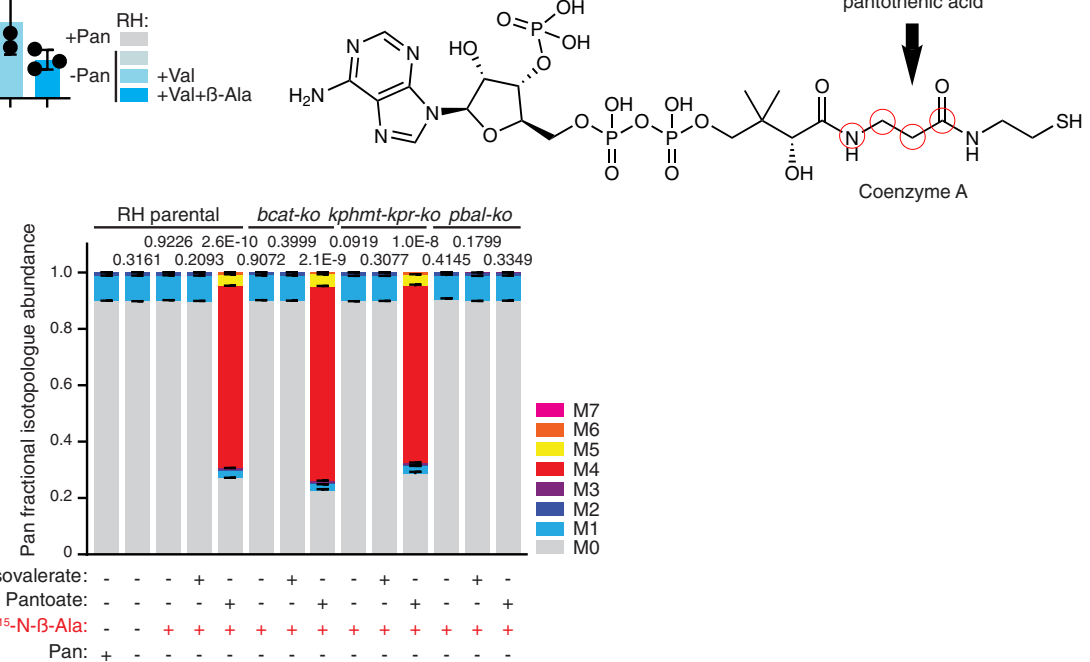

Fig. 6 T. gondii tachyzoites salvage Pan synthesis intermediates and use these for Pan synthesis. a Scheme of the Pan biosynthesis pathway, highlighting the ketopantoate hydroxymethyltransferase - ketopantoate reductase (KPHMT-KPR) and the pantoate beta alanine ligase (PBAL). Salvage of $\alpha$-ketoisovalerate and pantoate as precursors for Pan synthesis is shown. See Fig. 1 for abbreviations. b GC-MS Measurement of relative intracellular Pan levels in RH parental parasites following incubation in medium without Pan supplemented with valine (Val, $3 \mathrm{mM})$ and $\beta$-alanine $(\beta$-Ala, $2 \mathrm{mM})(n=3$, mean and SD, 2-sided Student's $t$ test). c Metabolite structures highlighting the theoretical incorporation of labeled atoms (red circles) from stable isotope labeled ${ }^{13} \mathrm{C}_{3} /{ }^{15} \mathrm{~N}$ - $\beta$-Ala into Pan and into $\mathrm{CoA}$. Exact masses of the parental ion (MO), and heavy stable isotope labeled (M4) are presented in black and red, respectively. $\mathbf{d}$ LC-MS analysis of the relative isotopologue (MO, M4) abundance of RH parental derived metabolites following incubation in medium with $3 \mathrm{mM}{ }^{13} \mathrm{C}_{3} /{ }^{15} \mathrm{~N}$ - $\beta$-Ala or $100 \mu \mathrm{M}{ }^{13} \mathrm{C}_{3} /{ }^{15} \mathrm{~N}$-Pan ( $n=4$, mean and SD, 2-sided Student's $t$ test). e LC-MS analysis of Pan isotopologue (MO to M7) distribution of RH parental, bcat-ko, kphmt-kpr-ko, pbal-ko derived metabolites following growth over $40 \mathrm{~h}$ in regular DMEM, DMEM - Pan $+5 \%$ dialyzed FBS supplemented with metabolites as indicated: $\alpha$-ketoisovalerate, pantoate, ${ }^{13} \mathrm{C}_{3} /{ }^{15} \mathrm{~N}-\beta$-Ala (each at $0.8 \mathrm{mM}$ ). Statistical analysis was carried out on the percentage of labeling shown in supplementary data $(n=3$, mean and SD, 2-sided Student's $t$ test). $n$ number of independent biological replicates. Source data are provided as a Source Data file.

penultimate Pan synthesis enzyme, KPHMT-KPR were bypassed by providing pantoate, also salvaged by the parasite (Supplementary Fig. $7 \mathrm{c}$ ), and ${ }^{13} \mathrm{C}_{3} /{ }^{15} \mathrm{~N}-\beta$-Ala (both $0.8 \mathrm{mM}$ ) to the culture medium. Remarkably, provision of these two Pan precursors led to the formation of labeled Pan in all strains except pbal-ko (Fig. 6e, Supplementary Fig. 8a), providing clear evidence that $T$. gondii PBAL is functional and active. In conclusion, KPHMT$\mathrm{KPR}$ is an inactive enzyme in cultured T. gondii tachyzoites, preventing the de novo synthesis of Pan from Val and $\beta$-Ala.

Aside from the availability of precursors, Pan synthesis in $E$. coli is also regulated through inhibition of KPHMT activity by accumulation of downstream metabolites, including Pan and $\mathrm{CoA}^{46}$. It is plausible that a similar regulation at the level of the KPHMT-KPR enzyme is occurring in T. gondii and given that the enzyme is expressed, posttranslational modifications might regulate its activity. In conclusion, despite expressing the enzymes required for Pan biosynthesis, at least two of which are enzymatically active (BCAT: ${ }^{32}$, PBAL: this study), tachyzoites do not synthesize Pan unless the final precursors are added in the culture media, even when exogenous Pan levels are low. Thus, the physiological role of Pan synthesis is unclear and was further scrutinized as outlined below.

De novo Pan synthesis is dispensable and inactive in in vitro differentiated bradyzoites. To address why coccidian parasites, but not other Apicomplexa, have retained the capacity to produce Pan, its importance was examined in the cyst-forming bradyzoite stage. While type I RH parasites are highly virulent and cause acute infection, with mice typically succumbing to the infection within 7 days, type II parasites are less virulent, and infected mice commonly overcome the acute phase of infection and develop chronic toxoplasmosis. Of relevance, stage conversion from tachyzoites to bradyzoites can also be achieved in vitro, using stress triggers such as high $\mathrm{pH}^{47}$ on $T$. gondii strains that are prone to encystation (ME49, Pru), or by upregulating a recently identified bradyzoite differentiation factor, BFD1 ${ }^{48}$. Type II parasites, like the ME49 strain, display a tendency to convert spontaneously to bradyzoites at low levels even under standard culture conditions and have a slower growth rate than type I RH parasites.

To understand the role of Pan synthesis (Fig. 7a) in type II parasites, KPHMT-KPR-SMmyc and PBAL-Ty strains were generated in ME49 parasites, using the same strategy as in Fig. 5. The level of both proteins dropped significantly following conversion to bradyzoites through cultivation in alkaline medium for 7 days (Fig. 7b, quantified in 7c). Efficient tachyzoite to bradyzoite conversion was confirmed by detection of the tachyzoite marker surface antigen 1 (SAG1) and/or the late bradyzoite marker $\mathrm{P} 21^{49}$. While these reduced protein levels may indicate reduced activity of the pathway, it is unclear if protein levels correspond to enzymatic activity. Thus, the importance of 
a

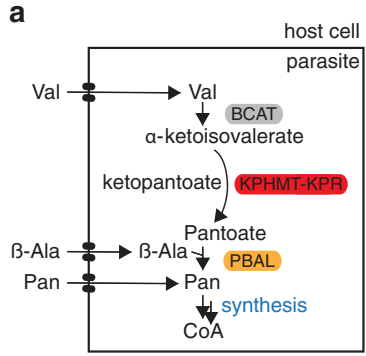

f

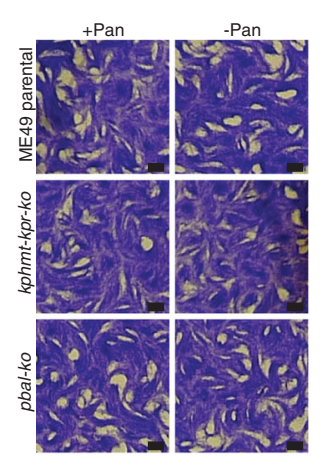

j

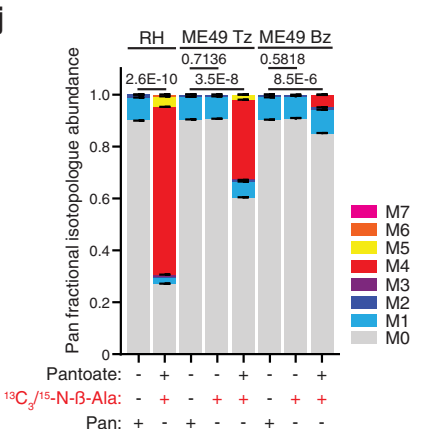

b

g
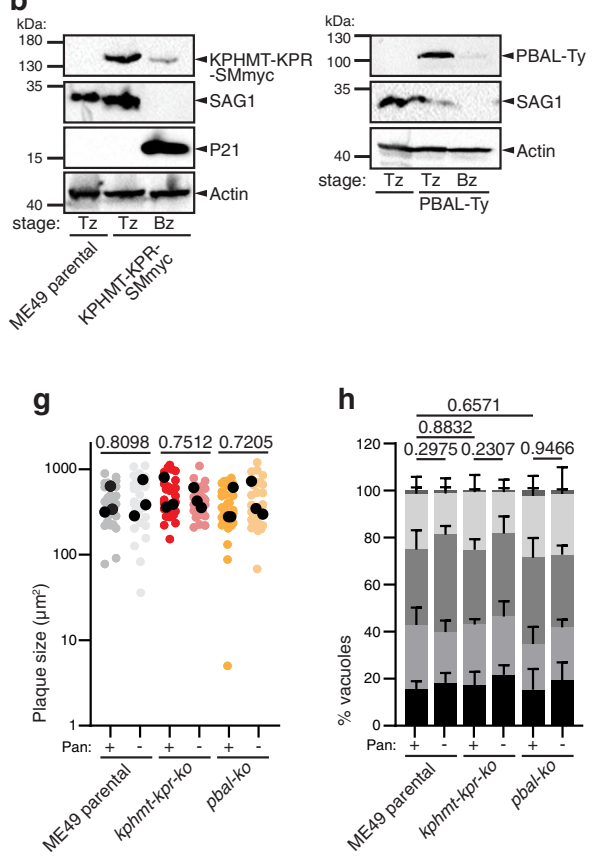

h

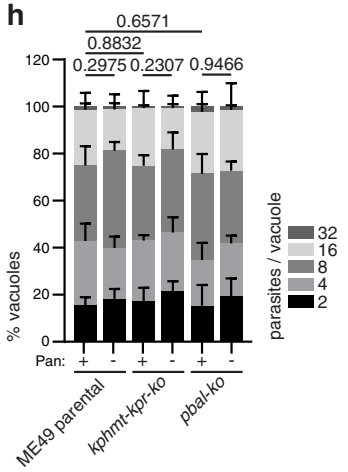

$\mathbf{k}$
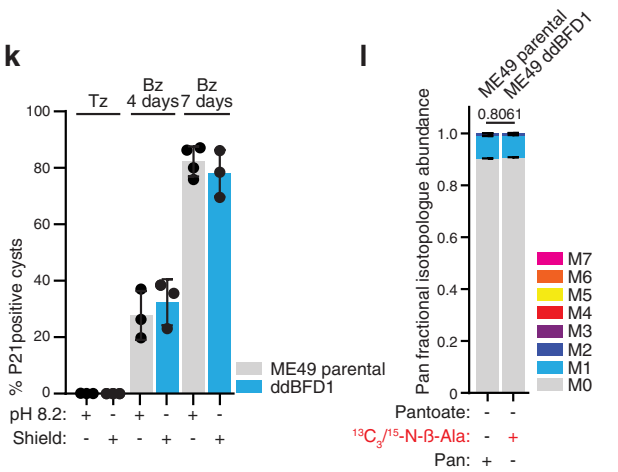

c

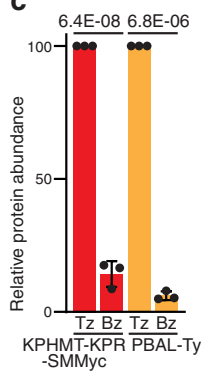

i

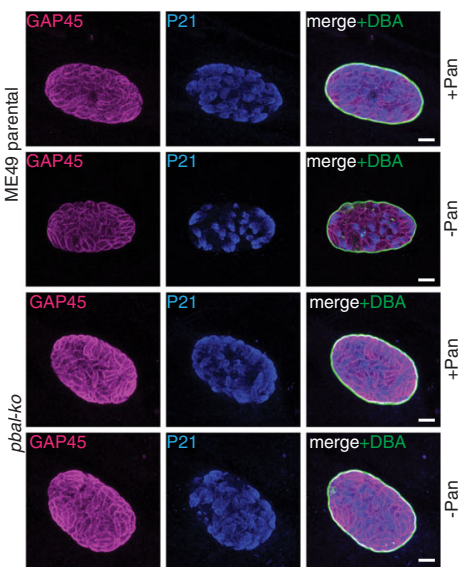

d

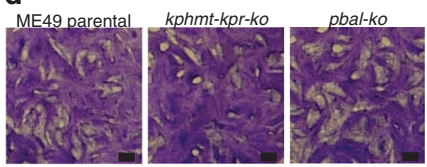

Plaque size $\left(\mu \mathrm{m}^{2}\right)$

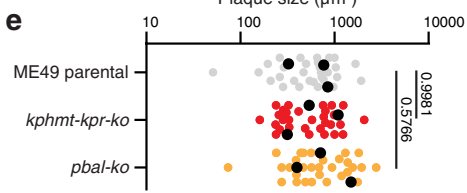

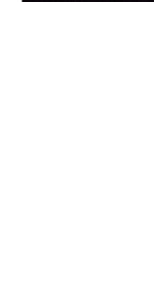

\section{$+\infty$}

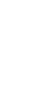

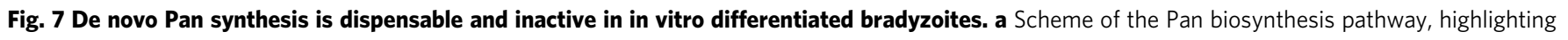
the ketopantoate hydroxymethyltransferase - ketopantoate reductase (KPHMT-KPR) and the pantoate beta alanine ligase (PBAL). See Fig. 1 for

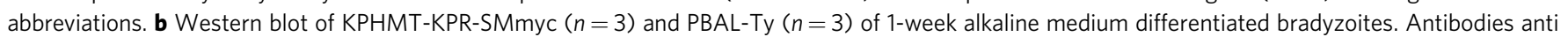

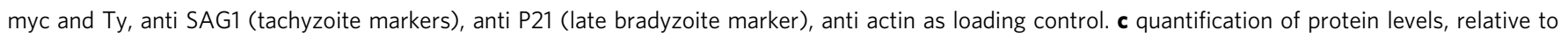

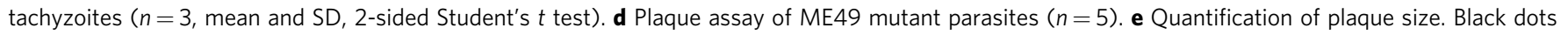

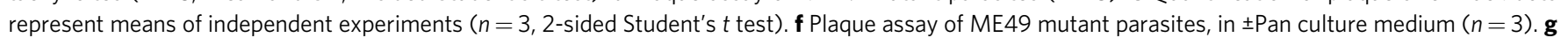

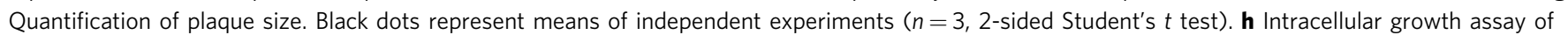

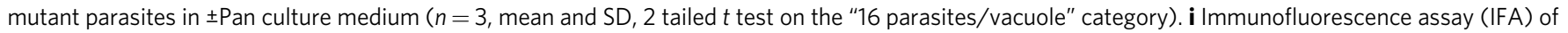

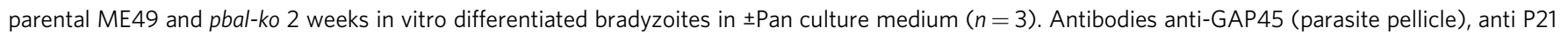

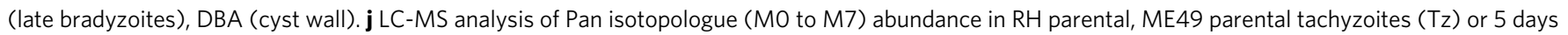

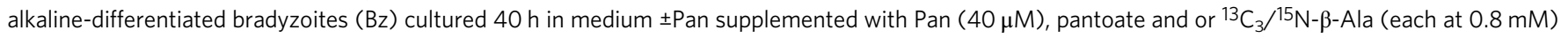

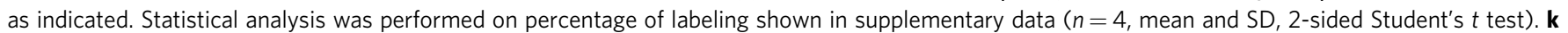

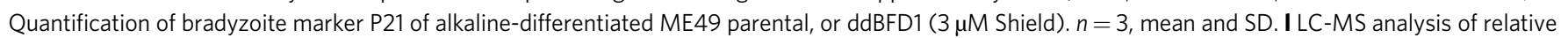

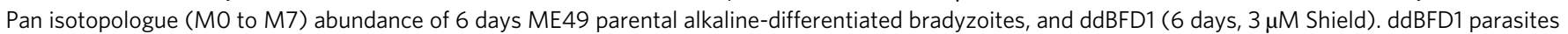
were cultured in medium containing ${ }^{13} \mathrm{C}_{3} /{ }^{15} \mathrm{~N}-\beta$-Ala $(0.8 \mathrm{mM}, 40 \mathrm{~h})$. Statistical analysis was carried out on the percentage of labeling shown in the

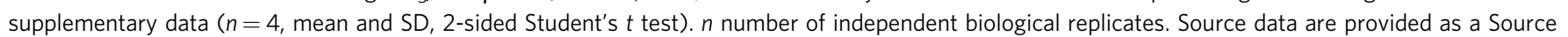
Data file. White scale bar $6 \mu \mathrm{m}$, black scale bar $1 \mathrm{~mm}$.

the pathway was probed further by generating $k p h m t-k p r-k o$ and pbal-ko mutants in the ME49 hxgprt-ko ku80-ko strain, referred to as ME49 parental (Fig. 7a, Supplementary Fig. 9a). By plaque assay in standard growth conditions, pbal-ko and kphmt-kpr-ko showed no lytic cycle defect when compared to the parental ME49 strain (Fig. 7d, e), as documented with RH (Fig. 5d). Furthermore, no plaque assay, or growth defect was detected when wildtype or mutant parasites were grown in -Pan medium (Fig. $7 \mathrm{f}-\mathrm{h}$ ). Given that no plaque assay defect could be detected, no defects are expected in any of the steps of the parasite lytic cycle. These results are consistent with those described for RH parasites above and point towards inactivity of the pathway in vitro, with parasites relying on highly efficient Pan salvage from the host, which supports growth even when Pan is removed from the culture medium.

To specifically address the importance of the pathway in encysted bradyzoites, ME49 parental and pbal-ko parasites were differentiated in alkaline medium, with or without Pan, for 2 weeks and cyst morphology was assessed by IFAs. The T. gondii cyst wall is rich in carbohydrates and efficiently stains with 
Dolichos biflorus agglutinin (DBA), while individual bradyzoites can be stained with the late bradyzoite marker P21. Neither parental ME49 parasites, nor pbal-ko displayed a defect in cyst morphology or in tachyzoite to bradyzoite differentiation when cultured in the presence or absence of Pan (Fig. 7i).

To measure whether Pan synthesis is active in bradyzoites in vitro, stable isotope labeling followed by LC-MS analysis was performed on parasite extracts collected 6 days after $\mathrm{pH}$ induced differentiation. As for type I RH parasites, no Pan synthesis in type II tachyzoites or bradyzoites was observed, as judged by the absence of labeled atoms incorporated from ${ }^{13} \mathrm{C}_{3} /{ }^{15} \mathrm{~N}-\beta$-Ala into Pan (Fig. 7j, Supplementary Fig. 8b). The same result was also obtained through measuring by GC-MS the absence of labeling in Pan of ME49 tachyzoites following incubation of parasites in medium containing $2 \mathrm{mM}{ }^{13} \mathrm{C}_{3} /{ }^{15} \mathrm{~N}-\beta$-Ala for $40 \mathrm{~h}$ (Supplementary Fig. 8c). To assess whether Pan synthesis occurs in ME49 parasites, when these are supplemented with pantoate and ${ }^{13} \mathrm{C}_{3} /$ ${ }^{15} \mathrm{~N}-\beta$-Ala, as described above for RH, ME49 parental tachyzoites and bradyzoites were cultured in the presence of $0.8 \mathrm{mM}$ pantoate in addition to $0.8 \mathrm{mM}{ }^{13} \mathrm{C}_{3} /{ }^{15} \mathrm{~N}-\beta$-Ala for $40 \mathrm{~h}$. As for $\mathrm{RH}$ parasites, these conditions led to Pan synthesis, highlighting that PBAL is also active in ME49 parasites. Strikingly, the Pan labeling was markedly reduced in ME49 tachyzoites and further in bradyzoites compared to $\mathrm{RH}$ parasites, labeled under equivalent conditions (Fig. 7j, data from Fig. $6 \mathrm{f}$ is included for comparison, Supplementary Fig. 8b). This is likely due to one or several of the following factors: reduced uptake of Pan precursors, reduced growth rate and overall metabolic activity, as well as reduced expression of $P B A L$ at the mRNA (Fig. 1a, Supplementary Data 1) and protein level (Fig. $7 \mathrm{~b}, \mathrm{c}$ ). To assess whether a different mode of differentiation would reveal a role for Pan synthesis, we made use of a strain that overexpresses the bradyzoite differentiation factor BFD1, which was recently identified ${ }^{48}$. The bradyzoite differentiation factor was fused to a destabilization domain (ddBFD1), which is stabilized in the presence of the ligand Shield. Efficient differentiation of ddBDF1+Shield parasites, comparable to that through alkaline $\mathrm{pH}$-treatment of parental ME49, was validated by assessing the P21 staining (Fig. 7k).

Similar to when parasites are differentiated through alkaline $\mathrm{pH}$, Pan synthesis remained inactive in ddBFD1+Shield, as judged by the absence of labeling in Pan when parasites were cultured in the presence of ${ }^{13} \mathrm{C}_{3} /{ }^{15} \mathrm{~N}-\beta$-Ala $(0.8 \mathrm{mM})$ for $40 \mathrm{~h}$ (Fig. 7l, Supplementary Fig. 8d). In summary, Pan synthesis was found to be dispensable and inactive in in vitro ME49 tachyzoites and bradyzoites, as observed for $\mathrm{RH}$ tachyzoites in culture.

Pantothenate synthesis is essential for chronic infection of $T$. gondii in vivo. Lastly, the relevance of Pan biosynthesis (Fig. 8a) was investigated in vivo during acute and chronic infection in the mouse model of toxoplasmosis. RH kphmt-kpr-ko and pbal-ko strain mutants exhibited the same virulence during acute infection of mice, as compared to the parental strain, with all mice succumbing to the infection within 10 days (Fig. 8b).

To address the function of PBAL more specifically, ME49 pbal$k o$ parasites were complemented with either a C-terminally myctagged full length cDNA of T. gondii PBAL (pbal-ko::cTgPBALmyc), or a C-terminally myc-tagged E. coli PBAL enzyme (pbal$k o:: c E c$ PBAL-myc) (Supplementary Fig. 8b). Both constructs were under the control of the constitutive tubulin promoter. Western blot analysis showed the expected MW for both tagged proteins (Fig. 8c). While IFAs showed nuclear localization for TgPBALmyc, consistent with its endogenous localization, EcPBAL-myc was found to be cytosolic (Fig. 8d). To ensure that these strains were not impacted by the complementation, plaque assays were performed under standard growth conditions and no defect or gain of fitness was associated with the complemented strains (Fig. 8e, f). Finally, virulence of the panel of mutants was tested during chronic infection in mice. While ME49 parasites are less virulent compared to $\mathrm{RH}$, and mice usually survive the acute phase of the disease, in some cases, mice succumb to the infection over the course of the experiment. This may be owed to an intense acute infection (first two weeks following inoculation), or a severe chronic infection (3-4 weeks after infection) associated with very high cyst numbers that can lead to weight loss, loss of motor skills and apathy. The kphmt-kpr-ko and pbal-ko mutants in ME49 showed a significant reduction in virulence compared to mice infected with the ME49 parental strain, which partially (40 $\%$ by day 30 ) succumbed to the infection over the course of the experiment, many as late as 3-4 weeks after inoculation (Fig. 8g). Five weeks after infection, the surviving mice were sacrificed to determine the cyst burden in the brain. Strikingly, the absence of either KPHMT-KPR or PBAL led to a dramatic drop in cyst numbers in the chronically infected animals (Fig. 8h) including a mouse with zero cysts infected with pbal-ko parasites, which was confirmed to be infected by testing for seroconversion (Fig. $8 \mathrm{~h}$, Supplementary Fig. 9c). Complementation of pbal-ko with either TgPBAL-myc or EcPBAL-myc led to a minor rescue of virulence, and an incomplete but significant restoration of cyst numbers, demonstrating the activity and essentiality of Pan synthesis in the bradyzoite stage (Fig. $8 \mathrm{~h}$ ). The virulence of the complementing strains in the mice is expected to be reduced with respect to ME49 parental strain, given the only partial complementation of the cyst burden. The few cysts produced during infection with kphmt-kpr$k o$ and pbal-ko were collected and shown to recrudesce in vitro by infecting monolayers of HFFs within 2 weeks, to levels comparable to parental ME49 (Fig. 8i), indicating that remaining cysts are viable and can access sufficient Pan from the host cell.

While the Pan synthesis pathway was at least partially inactive in vitro, hampering de novo Pan synthesis, the in vivo model for the chronic stage of disease uncovers a function for the complete pathway during encystation. This specific role of Pan synthesis is consistent with conservation of the pathway exclusively in coccidia amongst apicomplexans.

Taken together, the findings highlight a discrepancy between the metabolism of in vitro and in vivo differentiated bradyzoites, with Pan synthesis being dispensable in vitro but critical for cyst formation in vivo (Fig. 8j). Although in vitro differentiated bradyzoites recapitulate several hallmarks of in vivo bradyzoites such as the formation of cyst wall, accumulation of amylopectin granules and expression of several marker proteins within the plasma membrane (SRS9, PMA1) and in the cytoplasm (LDH2, BAG1 $)^{50}$, little is known about how closely the metabolism of in vitro bradyzoites resembles that of those in vivo. Undoubtedly, the culture in rich medium at ambient $\mathrm{O}_{2}$ in human foreskin fibroblasts, provides a suboptimal model for the conditions encountered by bradyzoites within the brain. Current metabolomic approaches do not allow the measurement of pure in vivo bradyzoites as they are hampered by low parasite yields, host cell/ tissue contamination and long purification protocols, which are expected to result in a distortion of the metabolome.

It remains unclear whether the switch to synthesize Pan occurs specifically in response to sensing altered levels of metabolites, such as Pan and $\beta$-Ala, or whether it occurs as part of a larger transcriptional regulatory switch following differentiation in vivo. Pan concentrations may vary in different regions of the brain ${ }^{51}$, likely making Pan synthesis essential in only a fraction of the developing cysts. Exogenous Pan is expected to cross the parasite's parasitophorous vacuole membrane and the cyst wall through the molecular sieve $(<1 \mathrm{kDa}$ and $1.3 \mathrm{kDa})$, for which the exclusion size is well over the MW of Pan ${ }^{52,53}$. Nevertheless, how parasites within mature cysts access host metabolites remains 
a

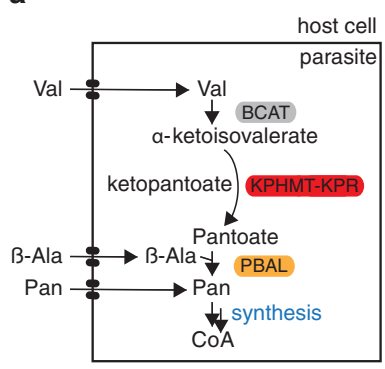

b

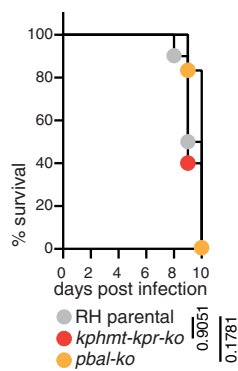

g

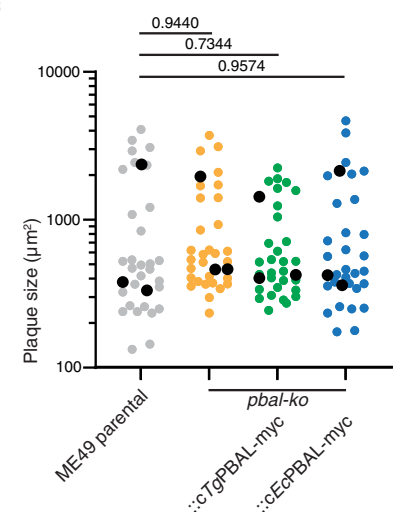

C

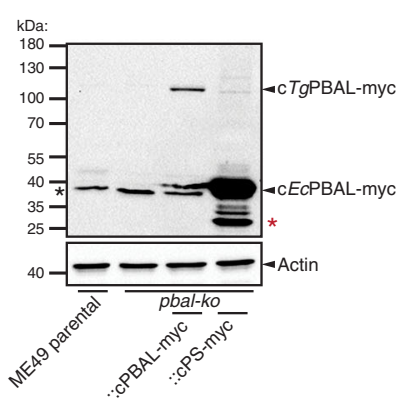

h

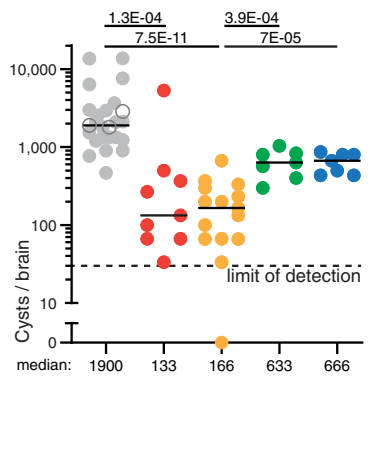

d

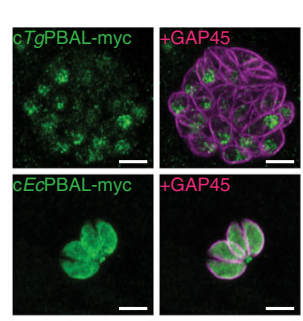

e

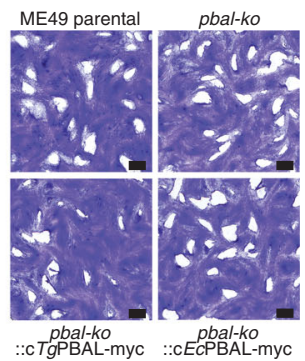

i

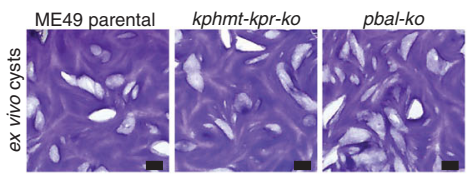

j

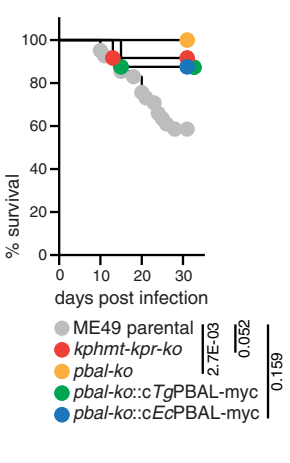

ME49 parental

- kphmt-kpr-ko

pbal-ko

pbal-ko::cTgPBAL-myc

pbal-ko::cEcPBAL-myc

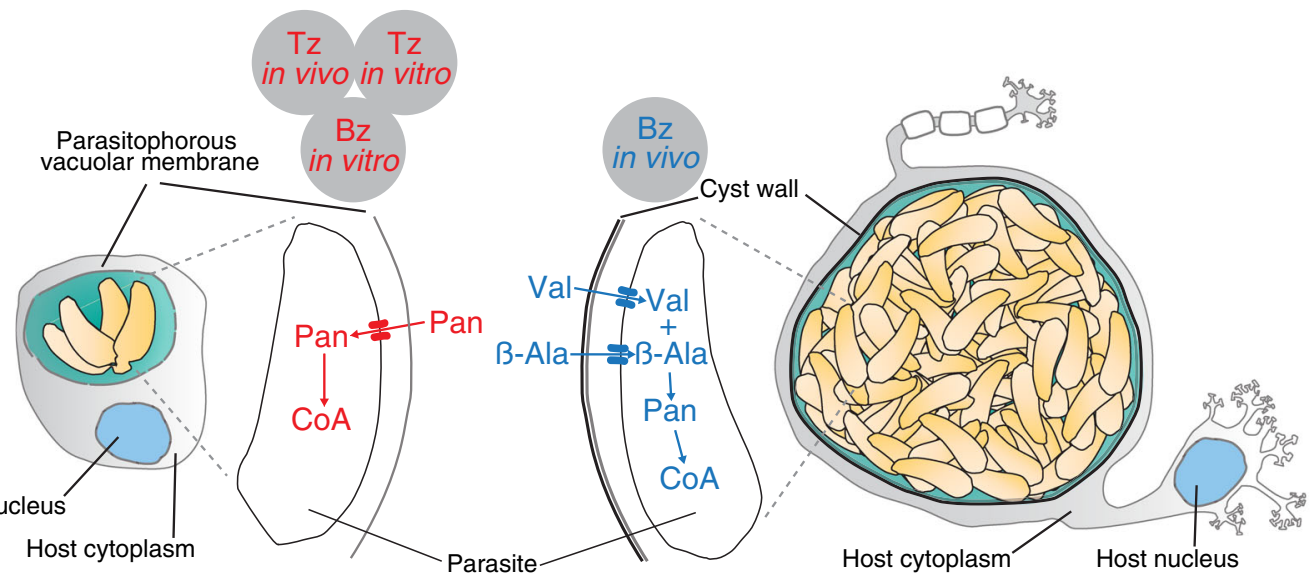

Fig. 8 Pantothenate synthesis is essential for chronic infection of T. gondii in vivo. a Scheme of the Pan biosynthesis pathway, highlighting the ketopantoate hydroxymethyl transferase - ketopantoate reductase (KPHMT-KPR) and the pantoate beta alanine ligase (PBAL). See Fig. 1 for abbreviations. b Survival curve of mice infected with RH parental $(n=10), k p h m t-k p r-k o(n=5)$ and pbal-ko $(n=5)$ parasites (from 2 experiments, logrank Mantel-Cox test). c Western blot of complementant strains pbal-ko::cTgPBAL-myc $(n=3)$ and pbal-ko::cEcPBAL-myc $(n=4)$. Antibodies anti myc, anti actin as loading control. d IFA of pbal-ko::cTgPBAL-myc $(n=5)$ and pbal-ko::cEcPBAL-myc $(n=3)$. Antibodies anti myc, anti GAP45 as marker of parasite pellicle. e Plaque assay of ME49 parental, pbal-ko and complement strains::cTgPBAL-myc,::cEcPBAL-myc $(n=3)$. $\mathbf{f}$ Quantification of plaque size. Black dots present mean values of independent experiments $(n=3)$. g Survival curves of mice infected with ME49 parental $(n=41,24$ survived), ME49 kphmt-kpr-ko ( $n=12$, 11 survived), ME49 pbal-ko ( $n=17,17$ survived), ME49 pbal-ko::cTgPBAL-myc ( $n=8,7$ survived), ME49 pbal-ko::cEcPBAL-myc ( $n=8,7$ survived) strains, pooled from 5 independent experiments. h Ex vivo cyst count from mouse brains infected for 5 weeks with parental ME49, kphmt-kpr-ko, pbal-ko, pbalko:::cTgPBAL-myc, and pbal-ko:::cEcPBAL-myc parasites ( $n=25,9,15,7$ and 7, respectively, pooled from 5 independent experiments). Empty circles present counts before the 5 weeks endpoint. Median is represented, 2-sided Mann-Whitney test. i Representative plaque assay of brain-derived cysts from ME49 parental, kphmt-kpr-ko and pbal-ko infected mice $(n=3,3,4$, respectively). $\mathbf{j}$ Cartoon schematic depicting the different modes of Pan acquisition in tachyzoites (Tz) and bradyzoites (Bz). $n$ number of independent biological replicates. Source data are provided as a Source Data file. White scale bar $5 \mu$ m. Black scale bar $1 \mathrm{~mm}$. Red asterisk marks degradation product of tagged protein. Black asterisk marks unspecific signal.

unknown. The average $\beta$-Ala concentration within the brain, the main site of $T$. gondii persistence, has been estimated to be $30-80 \mu \mathrm{M}^{54,55}$, hence it is sufficiently abundant for salvage by $T$. gondii bradyzoites. Intriguingly, $\beta$-Ala is particularly abundant in tissues that function as primary niches for T. gondii bradyzoite cysts during chronic toxoplasmosis, such as the central nervous system (CNS), the retina and muscle tissue ${ }^{55}$, possibly making it an essential metabolite for encystation. It is compelling to speculate that $\beta$-Ala in these tissues may be a factor contributing to the tissue tropism of T. gondii bradyzoites.

The identification of a metabolic pathway that is essential for the persistent stage of toxoplasmosis but is absent in its host paves the way for the development of new intervention. Of interest, an M. tuberculosis strain auxotrophic for Pan also 
presents a dramatically reduced virulence in the mouse model ${ }^{56}$, indicating that Pan synthesis could be an essential feature for chronic infection of various intracellular pathogens.

\section{Methods}

Parasite strains, culture and selection. RH hxgprt-ko ku80-ko parasites (RH) ${ }^{57}$, RH ku80-ko hxgprt-ko::Tir1 ${ }^{14}$, and ME49 hxgprt-ko ku80-ko (ME49) were grown in confluent human foreskin fibroblasts (HFF, ATCC SCRC-1041) in DMEM (Gibco 41965039) supplemented with $5 \%$ fetal calf serum and $40 \mu \mathrm{g} / \mathrm{ml}$ gentamycin (Gibco 15710-049). Parasites were selected for 1 week with $25 \mu \mathrm{g} / \mathrm{ml}$ mycophenolic acid and $50 \mu \mathrm{g} / \mathrm{ml}$ xanthine for HXGPRT positive selection ${ }^{58}, 1 \mu \mathrm{M}$ pyrimethamine for DHFR-TS positive selection ${ }^{59}, 5 \mu \mathrm{M}$ FUDR for UPRT negative selection ${ }^{60}$. Stable parasite populations were cloned by serial dilution.

Pantothenate depletion and media supplementations. Custom-made pantothenate depleted medium was purchased from Gibco based on regular DMEM recipe (Gibco 41965039). 5\% dialyzed FBS (Pan Biotech P30-2102; 10,000 Da exclusion size membrane) and $40 \mu \mathrm{g} / \mathrm{ml}$ gentamycin (Gibco 15710-049) were supplemented. HFFs were grown for at least one day in Pan-depleted medium prior parasite infection. Before medium change, HFFs were washed once with Pan depleted media to remove residual Pan. Regular DMEM + 5\% FCS was supplemented with indicated concentrations of additional pantothenate (Enzo ALX-460003-G011), PanSH (Sigma 16702) or CoA (Sigma C3144) for experiments described in Fig. 31, m.

Cloning and parasite lines generation. Primers are listed in Supplementary Table 1. PanK1-mAID-HA parasites were generated with primers 9157 (gRNA $^{12,13}$ ), 9158/9159 (homology template ${ }^{14}$ ); integration was checked with primer couples 9160/5370, 1629/9161, 9160/9161. PanK1-Ty parasites were generated with primers 9157 (gRNA), 9266/9267 (homology template containing Tytag). PanK2-mAID-HA parasites were generated with primers 9162 (gRNA), 9163/ 9164 (homology template); integration was checked with primer couples 9165/ 7081, 4609/9263, 9165/9263. PanK2-Ty were generated with primers 9162 (gRNA), 9268/9269 (homology template). PPCDC-mAID-HA parasites were generated with primers 7897 (gRNA), 7798/7799 (homology template); integration was checked with primer couples 6255/7081, 1629/7726, 6255/7726. PPCDC-Ty parasites were generated by cloning KpnII/NsiI the 6253/6254 PCR amplified 3' of the PPCDC gene in $\mathrm{p} 3 \mathrm{xTy}-\mathrm{HX}$ plasmid, linearized in the PPCDC sequence and transfected. DPCK-iKD parasites were generated with primers 7960 (gRNA), 8247/8256 (homology template ${ }^{61}$ ); integration was checked with primer couples 8249/3596, $8250 / 1935,8249 / 8250$. DPCK-SMmyc were generated with primers 8251 (gRNA), 9153/9154 (homology template ${ }^{24}$ ). DPCK cDNA was generated with primers 9046/ 9047 and cloned EcoRI/EcoRV in a modified pT8/UPRT plasmid ${ }^{60}$, linearized NruI/ApaI, cotransfected with a gRNA targeting the UPRT locus ${ }^{13}$ to generate the cDPCK-Myc parasite line. PBAL-Ty parasites were generated by cloning ApaI/SpfI the 7793/7794 PCR amplified 3' of the PBAL gene in p3xTy-HX plasmid, linearized NsiI and transfected. pbal-ko parasites were generated with primers $7228 / 7229$ $\left(2 \mathrm{gRNA}^{62}\right), 7230 / 7231$ (homology template); integration was checked with primer couples 7232/2074, 1629/7233, 7232/7628. KPHMT-KPR-SMmyc parasites were generated with primers 9200 (gRNA), 9381/9382 (homology template). Kphmtkpr-ko parasites were generated with primers 9374/9375 (2gRNA), 9376/9377 (homology template); integration was checked with primer couples 9378/2018, 2017/ 9379, 9378/9380. PBAL full length cDNA (PCR amplified 10121/9156) and E. coli PBAL (PCR amplified 10126/10127) were cloned EcoRI/EcoRV in pT8/ UPRT. The plasmids were linearized AatII/KpnI and cotransfected with a gRNA targeting the UPRT locuc. Integrations of the constructs in the UPRT locus were checked with primers $8414 / 3979$, while loss of the locus with $9318 / 9319$. Integration PCRs were performed once for each strain. Strains involved in animal experimentation were additionally tested for the correct genotype before every experiment.

Immunofluorescence assays (IFAs). Parasites grown in HFF on coverslips were fixed with $4 \%$ paraformaldehyde $0.05 \%$ glutaraldehyde for 15 min. Fixative was quenched with PBS $0.1 \mathrm{M}$ glycine. Coverslips were permeabilized $10 \mathrm{~min}$ with PBS $0.2 \%$ Triton X-100, blocked $1 \mathrm{~h}$ with blocking buffer (PBS $0.2 \%$ Triton X-100 2\% bovine serum albumin), incubated with primary and secondary antibodies in blocking buffer. Images were acquired with a confocal microscope (Zeiss LSM700). Images were processed with ImageJ.

Intracellular growth assays. Parasites were inoculated on coverslips with confluent HFF. Briefly centrifuged ( $1 \mathrm{~min}$ at $1100 \times g)$ and allowed to infect for $1 \mathrm{~h}$ at $37^{\circ} \mathrm{C}$. Non invaded parasites were removed by washing twice with DMEM. Coverslips were fixed for immunofluorescence $24 \mathrm{~h}$ post infection. Antibody staining (rabbit anti GAP45 and mouse anti GRA1) was used to quantify the number of parasites per vacuole, for a minimum of 200 vacuoles per condition. Quantification was performed blindly. Datasets were analyzed and plotted with GraphPad Prism.
Plaque assays. Freshly lysed parasites were inoculated on concluent HFF monolayers, supplemented or depleted, if indicated. Plaque sizes were quantified using the FindEdges and Segmentation (LevelSets) plugins in ImageJ (FIJI) software. Smaller dots represent each plaque size (10 for each independent experiment), while bigger black dots indicate the mean of each independent experiment.

Western blot and solubility assays. Parasite samples were lysed in Laemmli buffer with $10 \mathrm{mM}$ DTT, boiled $10 \mathrm{~min}$ and loaded in an SDS-page gel for electrophoresis in reducing conditions. Blots were transferred on nitrocellulose membranes, blocked and incubated with antibodies in blocking buffer (PBS 0.05\% Tween $205 \%$ non-fat milk). Freshly lysed parasites were collected, resuspended and freeze-thawed in either PBS, PBS $1 \%$ Triton X-100, PBS $1 \mathrm{mM} \mathrm{Na}_{2} \mathrm{CO}_{3}$, or $0.1 \%$ SDS. Soluble and insoluble fractions (sn and $\mathrm{p}$, respectively) were separated by centrifugation at $30,000 \times g$ after $30 \mathrm{~min}$ incubation on ice.

Co-immunoprecipitation. Freshly lysed parasites were collected, lysed in cold IP buffer (PBS, 0.5\% Triton X-100, protease inhibitor), freeze thawed 3 times, sonicated 5 times, centrifugated $30 \mathrm{~min}$ at $21,000 \times g$ at $4^{\circ} \mathrm{C}$. The supernatant was collected (a fraction kept as input sample) and incubated $2 \mathrm{~h}$ at $4{ }^{\circ} \mathrm{C}$ with mouse anti Ty ascite antibody or mouse anti HA antibody, $2 \mathrm{~h}$ with protein A sepharose beads. Centrifuged $2 \mathrm{~min}$ at $2000 \times g$ at $4^{\circ} \mathrm{C}$ (a fraction kept as wash sample), washed 3 times with IP buffer and resuspended in Laemmli buffer.

Antibodies. Polyclonal rabbit anti GAP45 $(1: 10,000)^{63}$, anti HA (1:1,000, Sigma H6908), anti HSP70 $(1: 1,000)^{64}$, anti CPN60 $(1: 3,000)^{65}$, anti-catalase $(1: 2,000)^{66}$ anti IMC1 $(1: 2,000)^{67}$. Monoclonal mouse anti actin $(1: 20)^{68}$, anti GRA1 and anti GRA3 (1:20, gifts of Dr. J. F. Dubremetz), anti myc (1:10, 9E10), anti Ty (1:10, BB2), anti P21 (1:10 $)^{49}$, anti SAG1 (1:10, gift of Dr. J. F. Dubremetz). FITC conjugated lectin (DBA, 1:500, Vector Laboratories FL-1031-2). Secondary antibodies for immunofluorescence: anti mouse Alexa fluor 405 (1:3000, Invitrogen A31553), 488 (1:3000, Invitrogen A11001), 594 (1:3000, Invitrogen A11005), anti-rabbit Alexa fluor 488 (1:3000, Invitrogen A11008), 594 (1:3000, Invitrogen A11012). Secondary antibodies for western blot: anti mouse HRP (1:3000, Sigma A5278), anti-rabbit HRP (1:3000, Sigma A8275).

Bradyzoite differentiation. In vitro bradyzoite differentiation was performed by culturing ME49 infected HFF in alkaline medium (RPMI 1640 (Gibco 51800-019), $50 \mathrm{mM}$ HEPES, $3 \%$ fetal calf serum, $40 \mu \mathrm{g} / \mathrm{ml}$ gentamycin (Gibco 15710-049), pH 8.2 with $\mathrm{NaOH}$ ) starting $24 \mathrm{~h}$ post infection ${ }^{47}$. Cultures were incubated at atmospheric $\mathrm{CO}_{2}$ concentration and medium was changed every 2 days. ME49 ddBFD1 strain was cultured in standard DMEM + 5\% FCS with $3 \mu \mathrm{M}$ Shield from day 1 post infection to differentiate into bradyzoites, as previously described ${ }^{48}$. Bradyzoite differentiation was quantified by immunofluorescence by counting P21 positive cysts $\left(\mathrm{P}_{2} 1^{+}, \mathrm{DBA}^{+}\right)$.

RNA extraction and reverse transcription. RNA was purified by acid phenolchloroform extraction and column purified (RNeasy mini kit QIAGEN REF 74104) from HFFs grown in media with or without Pan and/or infected with wild-type $\mathrm{RH}$ parasites (grown in regular or Pan-depleted media). All samples were collected in biological triplicate. Samples for Pan-depleted parasites were collected at passage 8 of Pan depletion. For cDNA generation RNA was treated with DNAse (QIAGEN REF 79254) and reverse transcribed (Thermo SuperscriptII Reverse transcriptase REF 18064014) following the manufacturer's instructions. For RNAseq the quantity and quality of the extracted RNA was measured with a Qubit Fluorometer (Life Technologies) and an Agilent 2100 BioAnalyser (Agilent Technologies) respectively. Ribosomal RNA was removed by applying polyA selection. RNA was subjected to $100 \mathrm{bp}$ single read sequencing on Illumina HiSeq 4000 (Illumina, San Diego, CA, USA) at the iGE3 Genomics platform at the University of Geneva (http://www.ige3.unige.ch/genomics-platform.php). Samples of uninfected and RH-infected HFFs (grown in media with or without Pan) were multiplexed and processed together in the sequencing lane of the flow cell.

RNaseq data processing and analysis. The quality of the reads was assessed with FASTX-Toolkit (http://hannonlab.cshl.edu/fastx_toolkit/). The Illumina adapter sequences from the raw reads and the poor-quality reads from the dataset were removed (parameters -q 30 and-length 36) with Trim Galore v 0.4 .2 (www.bioinformatics.babraham.ac.uk/projects/trim_galore). The resulting curated reads were aligned to the human reference genome (hg38_Ensembl) or T. gondii genome (TGGT1, toxodb.org v42) using HISAT2 aligner. Read counts on the genome features were generated using HTSeq-count (https://htseq.readthedocs.io/ en/release_0.11.1/count.html). The high-performance computing Baobab cluster at University of Geneva was used to perform all the computations. All samples were collected and processed in triplicates. Differences in gene expression were assessed using edgeR, a Bioconductor package in R (http://www.Rproject.org). Genes with at least a $\log \mathrm{FC}$ of \pm 0.5 (condition tested versus the WT) and an adjusted $p$ value (FDR) less than 0.05 were treated as differentially expressed, using standard statistical methods. A $t$-test was performed to obtain the $p$ value and the adjusted $p$ value (FDR) for each gene was calculated using the Benjamini-Hochberg method. 
Generally, the combination of the $\log \mathrm{FC}$ and FDR, represented in a 'volcano plot', is used to identify reliable and strong expression changes. The GeneIDs of the parasites (TGGT1) and the human host (HFFs), along with the fold-changes and p-values are summarized in Supplementary Data 2.

PantherDB (http://pantherdb.org/) was used to obtain the GO Terms for the up- and downregulated genes in HFFs (infected with RH parasites) grown in Pan depleted media (Supplementary Data 2, S2.2 and S2.3). To classify the GO Terms, we used the ENSEMBL gene IDs. "Homo sapiens" and "Functional classification viewed in gene list" were selected for the GO Terms and "Functional Classification viewed in graphic charts: Pie chart" was used for the charts. The distribution of the changes in the following GO Terms can be seen: Molecular Function, Biological Process, Cellular Component, Protein Class and Pathway. To observe the daughter branches of the parental GO Terms, the interactive and clickable online pie charts can be generated.

Animal experimentation. All animal experiments were conducted with the authorization numbers GE150-16 and GE121-19, according to the guidelines and regulations issued by the Swiss Federal Veterinary Office. No human samples were used for these experiments.

All animals were housed at the University of Geneva in room with day/night cycle of $12 \mathrm{~h} / 12 \mathrm{~h}$ and constant ambient temperature of $22^{\circ} \mathrm{C}$ and $35 \%$ humidity. Virulence assays were performed by intraperitoneal injection of 100 freshly lysed RH parasites in 7 weeks old female CD1 mice (Charles River). Mice were monitored daily and sacrificed at the onset of signs of acute infection (ruffled fur, difficulty moving, isolation). Cyst burden experiments were performed by intraperitoneal injection of 200 freshly lysed ME49 parasites into 7-week-old female B6CBAf1 mice (Janvier labs). Mice were monitored daily and sacrificed at the onset of signs of infection (ruffled fur, difficulty moving, isolation). 5 weeks post infection surviving mice were sacrificed. Brains were collected and homogenized by sequential passaging in needles with gauge diameter of $18 \mathrm{~g}, 20 \mathrm{~g}$ and $23 \mathrm{~g}$ in $1 \mathrm{ml}$ sterile PBS. $10 \mu \mathrm{l}$ of brain homogenates were counted blindly in triplicate with an optical microscope to determine cyst burden.

Ex vivo cysts viability assay. Brains from infected mice were collected in sterile conditions. Cysts were extruded from neurons by sequential needle-passaging as described in the "in vivo experiments" section. Cysts from brain homogenate were counted and 25, 5 and 1 cysts (all dilution performed in technical duplicate) per mouse brain were inoculated on HFF monolayer in DMEM 5\%FCS, allowed to settle for $24 \mathrm{~h}$ and washed from cell debris. Parasites were allowed to grow for 2 weeks before fixation and crystal violet staining. Replicating parasites were detected in all inoculated wells.

Enzyme expression and purification. Bacterial protein expression and purification: PBAL coding sequence was PCR amplified from CDNA with primers 9975/ 9976 and cloned by Gibson assembly into a pET-modified vector containing an $\mathrm{N}$-terminal maltose binding protein (MBP) and a C-terminal TEV recognition sequence followed by a His10-TwinSTREP tag. Catalytic domain of PBAL (PBALcd) was selected based on sequence homology and comparison with published crystal structures (PDB: 2X3F), PCR amplified with primers 10166/10167. PBAL was expressed in Rosetta DE3 pLysS cells. From a preculture, $1.5 \mathrm{l}$ of cells were grown until reaching OD600 of 0.6 , maintained on ice for $1 \mathrm{~h}$ and induced by addition of $0.5 \mathrm{mM}$ IPTG. Protein expression was done for $15 \mathrm{~h}$ at $20^{\circ} \mathrm{C}$ in a shaking incubator. Cells were harvested by centrifugation at $3000 \times g$ for $12 \mathrm{~min}$ at $4{ }^{\circ} \mathrm{C}$. The pellet was resuspended in $60 \mathrm{ml}$ of lysis buffer ( $50 \mathrm{mM}$ Tris $\mathrm{pH} 8$, $150 \mathrm{mM} \mathrm{NaCl}, 3 \mathrm{mM} \beta$-mercaptoethanol), lysed by shear forces by passing the sample twice through the Microfluidizer at 15,000 psi. Soluble material was separated from membranes and cell debris by centrifugation at $35,000 \times g$ for $35 \mathrm{~min}$ at $4{ }^{\circ} \mathrm{C}$. Supernatant was syringe-filtered at $5 \mu \mathrm{m}$ and applied to a $5 \mathrm{ml}$ STREP-tactin XT Superflow (iba) column. Column was washed first with $40 \mathrm{ml}$ of lysis buffer supplemented with $250 \mathrm{mM} \mathrm{NaCl}$, then with $40 \mathrm{ml}$ of lysis buffer. Protein was digested on-column usin $0.3 \mathrm{mg}$ of TEV protease. Tag-cleaved protein was eluted from the column by applying $15 \mathrm{ml}$ of lysis buffer. Collected protein was concentrated and applied to a Superdex 200, 10/300 size-exclusion chromatography column equilibrated in PBS. Fractions containing MBP-PBALcd were pooled and concentrated to $500 \mu \mathrm{l}$ using AMICON 30 MWCO. Protein concentration was estimated by measuring absorbance at $250 \mathrm{~nm}$ using a NanoDrop (ThermoFisher Scientific)

Culture and cell harvest for MS experiments. T. gondii parasites were cultured in complete medium (DMEM, $+5 \%$ FCS) or in custom culture medium -Pan (DMEM - Pan, $+5 \%$ dialyzed FBS) supplemented with Val (Sigma, V6504), pantoate (Sigma, 16682), $\alpha$-ketoisovalerate (Sigma, 198994), $\beta$-Ala (Sigma, 146064) or stable isotope labeled metabolites: ${ }^{13} \mathrm{C}_{3} /{ }^{15} \mathrm{~N}-\beta$-Ala (Cambridge Isotope Laboratories, CNLM-3946) or ${ }^{13} \mathrm{C}_{3} /{ }^{15} \mathrm{~N}$-Pan (Cambridge Isotope Laboratories, CNLM-7964). Concentrations and durations as indicated for each experiment.

For parasite harvest, excess culture medium was gently aspirated off the monolayer of host cells with freshly egressing parasites and ice-cold PBS was added to quench the metabolism. All subsequent steps were carried out on ice or at $4{ }^{\circ} \mathrm{C}$ for centrifugations. Parasites were harvested by scraping the infected host-cell monolayers and passing host cells through $28 \mathrm{~g}$ needles ( $3 \mathrm{x}$ ) before removing hostdebris through filtration ( $3 \mu \mathrm{m}$ pore size, Millipore, TSTP04700). The flow-through was collected on ice and filters were rinsed with additional ice-cold PBS to maximize the yield of parasites. The subsequent centrifugation and washing steps were carried out swiftly at $4{ }^{\circ} \mathrm{C}$ to minimize metabolic activity. Parasites were counted using a Neubauer counting chamber and $10^{8}$ parasites were pelleted by centrifugation $\left(2200 \times g, 10 \mathrm{~min}, 4^{\circ} \mathrm{C}\right)$ and pellets washed with ice-cold PBS (3x) to remove residual medium. The pellets were immediately processed as described below or stored at $-80^{\circ} \mathrm{C}$ until metabolite extraction.

GC-MS sample preparation and analysis. Metabolites were extracted from cell pellets through addition of $50 \mu \mathrm{l}$ chloroform and vigorous vortexing, followed by $200 \mu \mathrm{lmethanol/ultrapure} \mathrm{water} \mathrm{(3:1,} \mathrm{containing} 5 \mu \mathrm{M}$ scyllo-inositol (Sigma I8132) as internal standard. Insoluble material was pelleted by centrifugation $\left(21,000 \times g, 6 \mathrm{~min}, 4^{\circ} \mathrm{C}\right)$ and the supernatant transferred to a new microtube containing $100 \mu \mathrm{l}$ ultrapure water. The polar and apolar phases were separated through centrifugation $\left(21,000 \times g, 6 \mathrm{~min}, 4^{\circ} \mathrm{C}\right)$. The polar phase was taken off and dried $(50 \mu \mathrm{l})$ in a glass vial insert suitable for mass spectrometry vials using a centrifugal evaporator. For GC-MS analysis, samples were methoximated by resuspending the dried extract in $20 \mu \mathrm{l}$ pyridine (Sigma 270970) containing methoxyamine-hydrochloride $(20 \mathrm{mg} / \mathrm{ml}$; room temperature, over-night, Sigma 226904) and trimethylsilylated shortly before analysis through addition of $20 \mu \mathrm{l}$ $\mathrm{N}$,O-bis(trimethylsilyl)trifluoroacetamide with $1 \%$ trimethylchlorosilane (BSTFATMCS, Cerilliant, Sigma B-023). The GC-MS instrumentation and settings (electron ionization mode) were as described in ${ }^{62}$. All metabolites were identified based on the retention time and mass spectra of authentic standards. 3-trimethylsylil(TMS)-Pan qualifier ions were: $\mathrm{m} / \mathrm{z} 201,291$ and 420, for 2-TMS-Val: $\mathrm{m} / \mathrm{z} 144,218$ 246 and for 3-TMS- $\beta$-Ala: $m / z$ 174, 248 and 290 (Supplementary Fig. 4a). Previously, a detailed GC-MS study of the 3-TMS-Pan derivative investigated its fragmentation during electron ionization demonstrating that the ion $\mathrm{m} / \mathrm{z} 420$ corresponds to $\left[\mathrm{M}-\mathrm{CH}_{3}\right]^{+}$, while ions $\mathrm{m} / z 291$ and $\mathrm{m} / z 201$ derive from complex rearrangements but contain the full backbone of the $\beta$-Ala fraction of $\operatorname{Pan}^{69}$. Relative concentrations of Pan were determined by quantifying abundance of the parental ion $\mathrm{m} / \mathrm{z} 201$ in unlabeled samples relative to the ion $\mathrm{m} / \mathrm{z} 318$ of the internal standard (scyllo-inositol) or the total ion chromatogram (TIC), as indicated. Labeling in parasite-derived Pan was determined by quantifying the isotopologue distribution for the ion $\mathrm{m} / \mathrm{z}$ 201. Parental ions and their isotopologues were quantified using Xcalibur (ThermoFisher Scientific), OpenChrom and Excel (Microsoft) software following correction for natural abundance ${ }^{70}$.

UPLC-MS sample preparation and analysis. Parasites $\left(10^{8}\right.$ cells $)$ were harvested as described above. Metabolites were extracted from cell pellets by adding $200 \mu \mathrm{l}$ acetonitrile/ultrapure water (4:1) and vortexing vigorously. Insoluble material was pelleted through centrifugation $\left(21,000 \times \mathrm{g}, 6 \mathrm{~min}, 4^{\circ} \mathrm{C}\right)$. For reversed-phase UPLC-MS experiments the metabolite extract was sequentially dried as described above and resuspended in $50 \mu \mathrm{l}$ ultrapure water. For HILIC UPLC-MS analyses, the metabolite extract was injected directly. For metabolite quantification experiments, $10 \mu \mathrm{M}{ }^{13} \mathrm{C}_{3} /{ }^{15} \mathrm{~N}$-Pan (Figs. $4 \mathrm{n}$ and $5 \mathrm{k}$ ) or $40 \mu \mathrm{M}{ }^{13} \mathrm{C}_{6} /{ }^{15} \mathrm{~N}$-isoleucine (Figs. $2 \mathrm{l}, 3 \mathrm{k}$, and $4 \mathrm{~m}$ ) were included in the extraction solvent as internal standard. Abundances were normalized to the intensity of the internal standard and presented relative to the control strains as indicated (normalized to relative abundance of 1). The retention time and fragmentation of each metabolite was determined using authentic standards.

Reversed-phase UPLC-MS analyses. were performed by parallel reaction monitoring (PRM) experiments using a ThermoFisher Scientific Q Exactive Plus coupled to an UltiMate 3000 RSLC, or by multiple reaction monitoring (MRM) experiments using either a Sciex QTRAP 3200 (Fig. 6d) or a Sciex QTRAP 6500 (Figs. 4n and 5k) coupled to an Agilent Technologies 1290 Infinity LC Series. For all above instrument setups, the UPLC column was a Cortecs T3 column $(150 \times 2.1 \mathrm{~mm} \times$ $1.6 \mu \mathrm{m}$ ) equipped with its $5 \mathrm{~mm}$ VanGuard cartridge (Waters). Mobile phase composition and gradient as well as MS conditions are given as supplementary information (Supplementary Methods).

HILIC UPLC-MS analyses. were performed by parallel reaction monitoring (PRM) experiments using a ThermoFisher Scientific Q Exactive Plus coupled to an UltiMate 3000 RSLC. The UPLC column was a BEH Amide column $(150 \times 2.1 \mathrm{~mm} \times 1.7 \mu \mathrm{m})$ equipped with its $5 \mathrm{~mm}$ VanGuard cartridge (Waters). Mobile phase composition and gradient elution conditions, as well as MS parameters are given as supplementary information (Supplementary Methods).

LC-MS data processing. XCalibur (ThermoFisher Scientific), Skyline ${ }^{71}$, PeakView and MultiQuant (SCIEX) software were used to process the data. Relative metabolite levels and labeling were quantified using Excel (Microsoft). Metabolite levels were determined by quantifying the area under the curve relative to the internal standard. For PRM experiments, the TIC trace was used for quantification, for FSPRM experiments, the full scan trace was used and for MRM experiments, the first transition was selected for quantification. For labeling analyses shown in Figs. 6d or 5f, the relative levels of M0 and M4 or M7 isotopologues were determined for each 
metabolite. For data shown in Figs. 6e and 7j, 1, all Pan isotopologues in the full scan were measured (NEG mode) and quantified using El-Maven Software ${ }^{72}$. The relative isotopologue distribution is given and labeling (\%) was quantified based on the isotopologue distribution following correction for natural abundance ${ }^{70}$.

Reporting summary. Further information on research design is available in the Nature Research Reporting Summary linked to this article.

\section{Data availability}

The authors declare that the data supporting the findings of this study are available within the paper and its supplementary information files. Source data are provided with this paper. RNA sequencing data is deposited on the European Nucleotide Archive (ENA) following the ENA guidelines. The files can be accessed and downloaded via the link: http://www.ebi.ac.uk/ ena/data/view/PRJEB43225. RNA analysis output: https://doi.org/10.17632/zyrz6dcc85.173 Metabolomics datasets can be found in the Yareta (Geneva) repository following the link ${ }^{74}$ below. Data are sorted by platform (GC-MS, LC-MS) and figure panel. All files are deposited as original files (RAW) and as generic file formats (mzML or mzXML): https://doi.org/ 10.26037/yareta:bvz6yrckafdrxmzgn5hpuumkue. Source data are provided with this paper

Received: 13 February 2021; Accepted: 3 January 2022;

Published online: 17 January 2022

\section{References}

1. Flegr, J., Prandota, J., Sovickova, M. \& Israili, Z. H. Toxoplasmosis-a global threat. Correlation of latent toxoplasmosis with specific disease burden in a set of 88 countries. PLoS One 9, e90203 (2014).

2. McAuley, J. B. Congenital toxoplasmosis. J. Pediatr. Infect. Dis. Soc. 3(Suppl 1), S30-35 (2014).

3. Weiss, L. M. \& Kim, K. The development and biology of bradyzoites of Toxoplasma gondii. Front. Biosci. 5, D391-405 (2000).

4. Nissapatorn, V. Toxoplasma gondii and HIV: a never-ending story. Lancet HIV 4, el46-e147 (2017).

5. Wang, Z. D. et al. Toxoplasma gondii Infection in immunocompromised patients: a systematic review and meta-analysis. Front. Microbiol. 8, 389 (2017).

6. Kloehn, J. et al. Multi-omics analysis delineates the distinct functions of subcellular acetyl-CoA pools in Toxoplasma gondii. BMC Biol. 18, 67 (2020).

7. Oppenheim, R. D. et al. BCKDH: the missing link in apicomplexan mitochondrial metabolism is required for full virulence of Toxoplasma gondii and Plasmodium berghei. PLoS Pathog. 10, e1004263 (2014).

8. Tymoshenko, S. et al. Metabolic needs and capabilities of Toxoplasma gondii through combined computational and experimental analysis. PLoS Comput. Biol. 11, e1004261 (2015)

9. Krishnan, A., Kloehn, J., Lunghi, M. \& Soldati-Favre, D. Vitamin and cofactor acquisition in apicomplexans: synthesis versus salvage. J. Biol. Chem. 295, 701-714 (2020).

10. Muller, S. \& Kappes, B. Vitamin and cofactor biosynthesis pathways in Plasmodium and other apicomplexan parasites. Trends Parasitol. 23, 112-121 (2007).

11. Hart, R. J. et al. Genetic characterization of Plasmodium putative pantothenate kinase genes reveals their essential role in malaria parasite transmission to the mosquito. Sci. Rep. 6, 33518 (2016).

12. Sidik, S. M., Hackett, C. G., Tran, F., Westwood, N. J. \& Lourido, S. Efficient genome engineering of Toxoplasma gondii using CRISPR/Cas9. PLoS One 9, e100450 (2014).

13. Shen, B., Brown, K. M., Lee, T. D. \& Sibley, L. D. Efficient gene disruption in diverse strains of Toxoplasma gondii using CRISPR/CAS9. MBio 5, e01114-01114 (2014)

14. Brown, K. M., Long, S. \& Sibley, L. D. Plasma Membrane Association by N-Acylation Governs PKG Function in Toxoplasma gondii. mBio 8, https:// doi.org/10.1128/mBio.00375-17 (2017)

15. Sidik, S. M. et al. A genome-wide CRISPR screen in Toxoplasma identifies essential apicomplexan genes. Cell 166, 1423-1435 e1412 (2016).

16. Song, W. J. \& Jackowski, S. Kinetics and regulation of pantothenate kinase from Escherichia coli. J. Biol. Chem. 269, 27051-27058 (1994).

17. Tjhin, E. T., Howieson, V. M., Spry, C., van Dooren, G. G. \& Saliba, K. J. A novel heteromeric pantothenate kinase complex in apicomplexan parasites. PLoS Pathog. 17, e1009797 (2021).

18. de Villiers, M., Barnard, L., Koekemoer, L., Snoep, J. L. \& Strauss, E. Variation in pantothenate kinase type determines the pantothenamide mode of action and impacts on coenzyme A salvage biosynthesis. FEBS J. 281, 4731-4753 (2014).

19. Di Meo, I. et al. Acetyl-4'-phosphopantetheine is stable in serum and prevents phenotypes induced by pantothenate kinase deficiency. Sci. Rep. 7, 11260 (2017).
20. Bushell, E. et al. Functional profiling of a Plasmodium genome reveals an abundance of essential genes. Cell 170, 260-272 e268 (2017).

21. Hart, R. J., Abraham, A. \& Aly, A. S. I. Genetic characterization of coenzyme a biosynthesis reveals essential distinctive functions during malaria parasite development in blood and mosquito. Front. Cell Infect. Microbiol. 7, 260 (2017).

22. Rana, A. et al. Pantethine rescues a Drosophila model for pantothenate kinaseassociated neurodegeneration. Proc. Natl Acad. Sci. USA 107, 6988-6993 (2010).

23. Srivastava, A. et al. Stage-specific changes in Plasmodium metabolism required for differentiation and adaptation to different host and vector environments. PLoS Pathog. 12, e1006094 (2016).

24. Hortua Triana, M. A. et al. Tagging of weakly expressed Toxoplasma gondii calcium-related genes with high-affinity tags. J. Eukaryot. Microbiol. 65, 709-721 (2018).

25. Swift, R. P., Rajaram, K., Liu, H. B. \& Prigge, S. T. Dephospho-CoA kinase, a nuclear-encoded apicoplast protein, remains active and essential after Plasmodium falciparum apicoplast disruption. EMBO J., e107247, https:// doi.org/10.15252/embj.2020107247.

26. Meissner, M., Brecht, S., Bujard, H. \& Soldati, D. Modulation of myosin A expression by a newly established tetracycline repressor-based inducible system in Toxoplasma gondii. Nucleic Acids Res. 29, E115 (2001).

27. Soldati, D. \& Boothroyd, J. C. A selector of transcription initiation in the protozoan parasite Toxoplasma gondii. Mol. Cell Biol. 15, 87-93 (1995).

28. Radke, J. B., Burrows, J. N., Goldberg, D. E. \& Sibley, L. D. Evaluation of current and emerging antimalarial medicines for inhibition of Toxoplasma gondii growth in vitro. ACS Infect. Dis. 4, 1264-1274 (2018).

29. Schalkwijk, J. et al. Antimalarial pantothenamide metabolites target acetylcoenzyme A biosynthesis in Plasmodium falciparum. Sci. Transl. Med. 11, https://doi.org/10.1126/scitranslmed.aas9917 (2019).

30. Summers, R. L. et al. Chemogenomics identifies acetyl-coenzyme A synthetase as a target for malaria treatment and prevention. Cell Chem. Biol., https:// doi.org/10.1016/j.chembiol.2021.07.010 (2021).

31. Leonardi, R. \& Jackowski, S. Biosynthesis of pantothenic acid and coenzyme A. EcoSal Plus 2, https://doi.org/10.1128/ecosalplus.3.6.3.4 (2007).

32. Limenitakis, J. et al. The 2-methylcitrate cycle is implicated in the detoxification of propionate in Toxoplasma gondii. Mol. Microbiol. 87, 894-908 (2013).

33. Mageed, S. N. et al. Pantothenic acid biosynthesis in the parasite Toxoplasma gondii: a target for chemotherapy. Antimicrob. Agents Chemother. 58, 6345-6353 (2014).

34. Shanmugasundram, A., Gonzalez-Galarza, F. F., Wastling, J. M., Vasieva, O. \& Jones, A. R. Library of Apicomplexan Metabolic Pathways: a manually curated database for metabolic pathways of apicomplexan parasites. Nucleic Acids Res. 41, D706-713 (2013).

35. Ottenhof, H. H. et al. Organisation of the pantothenate (vitamin B5) biosynthesis pathway in higher plants. Plant J. 37, 61-72 (2004).

36. Saliba, K. J. \& Kirk, K. $\mathrm{H}^{+}$-coupled pantothenate transport in the intracellular malaria parasite. J. Biol. Chem. 276, 18115-18121 (2001).

37. Divo, A. A., Geary, T. G., Davis, N. L. \& Jensen, J. B. Nutritional requirements of Plasmodium falciparum in culture. I. Exogenously supplied dialyzable components necessary for continuous growth. J. Protozool. 32, 59-64 (1985).

38. Augagneur, $Y$. et al. Identification and functional analysis of the primary pantothenate transporter, PfPAT, of the human malaria parasite Plasmodium falciparum. J. Biol. Chem. 288, 20558-20567 (2013).

39. Kehrer, J. et al. A putative small solute transporter is responsible for the secretion of G377 and TRAP-containing secretory vesicles during Plasmodium gamete egress and sporozoite motility. PLoS Pathog. 12, e1005734 (2016).

40. Hammoudi, P. M., Maco, B., Dogga, S. K., Frenal, K. \& Soldati-Favre, D. Toxoplasma gondii TFP1 is an essential transporter family protein critical for microneme maturation and exocytosis. Mol. Microbiol. https://doi.org/ 10.1111/mmi.13981 (2018).

41. Srinivasan, B. et al. Extracellular 4'-phosphopantetheine is a source for intracellular coenzyme A synthesis. Nat. Chem. Biol. 11, 784-792 (2015).

42. MacRae, J. I. et al. Mitochondrial metabolism of glucose and glutamine is required for intracellular growth of Toxoplasma gondii. Cell Host Microbe 12, 682-692 (2012).

43. Webb, M. E. \& Smith, A. G. Pantothenate biosynthesis in higher plants. Adv Botanical Res. 58, 203-255 (2011).

44. Kalyankar, G. D. \& Meister, A. Enzymatic synthesis of carnosine and related beta-alanyl and gamma-aminobutyryl peptides. J. Biol. Chem. 234, 3210-3218 (1959).

45. Dusch, N., Puhler, A. \& Kalinowski, J. Expression of the Corynebacterium glutamicum panD gene encoding L-aspartate-alpha-decarboxylase leads to pantothenate overproduction in Escherichia coli. Appl. Environ. Microbiol. 65 $1530-1539$ (1999).

46. Powers, S. G. \& Snell, E. E. Ketopantoate hydroxymethyltransferase. II. Physical, catalytic, and regulatory properties. J. Biol. Chem. 251, 3786-3793 (1976). 
47. Weiss, L. M. et al. A cell culture system for study of the development of Toxoplasma gondii bradyzoites. J. Eukaryot. Microbiol. 42, 150-157 (1995).

48. Waldman, B. S. et al. Identification of a master regulator of differentiation in Toxoplasma. Cell 180, 359-372 e316 (2020).

49. Soete, M., Fortier, B., Camus, D. \& Dubremetz, J. F. Toxoplasma gondii: kinetics of bradyzoite-tachyzoite interconversion in vitro. Exp. Parasitol. 76, 259-264 (1993).

50. Mayoral, J., Di Cristina, M., Carruthers, V. B. \& Weiss, L. M. Toxoplasma gondii: Bradyzoite Differentiation In Vitro and In Vivo. Methods Mol. Biol. 2071, 269-282 (2020).

51. Ismail, N. et al. Vitamin B5 (d-pantothenic acid) localizes in myelinated structures of the rat brain: potential role for cerebral vitamin $\mathrm{B} 5$ stores in local myelin homeostasis. Biochem Biophys. Res Commun. 522, 220-225 (2020).

52. Lemgruber, L., Lupetti, P., Martins-Duarte, E. S., De Souza, W. \& Vommaro, R. C. The organization of the wall filaments and characterization of the matrix structures of Toxoplasma gondii cyst form. Cell Microbiol 13, 1920-1932 (2011).

53. Schwab, J. C., Beckers, C. J. \& Joiner, K. A. The parasitophorous vacuole membrane surrounding intracellular Toxoplasma gondii functions as a molecular sieve. Proc. Natl Acad. Sci. USA 91, 509-513 (1994).

54. del Rio, R. M., Orensanz Munoz, L. M. \& DeFeudis, F. V. Contents of betaalanine and gamma-aminobutyric acid in regions of rat CNS. Exp. Brain Res. 28, 225-227 (1977).

55. Tiedje, K. E., Stevens, K., Barnes, S. \& Weaver, D. F. Beta-alanine as a small molecule neurotransmitter. Neurochem. Int. 57, 177-188 (2010).

56. Sambandamurthy, V. K. et al. A pantothenate auxotroph of Mycobacterium tuberculosis is highly attenuated and protects mice against tuberculosis. Nat. Med. 8, 1171-1174, https://doi.org/10.1038/nm765 (2002).

57. Huynh, M. H. \& Carruthers, V. B. Tagging of endogenous genes in a Toxoplasma gondii strain lacking Ku80. Eukaryot. Cell 8, 530-539 (2009).

58. Donald, R. G., Carter, D., Ullman, B. \& Roos, D. S. Insertional tagging, cloning, and expression of the Toxoplasma gondii hypoxanthine-xanthineguanine phosphoribosyltransferase gene. Use as a selectable marker for stable transformation. J. Biol. Chem. 271, 14010-14019 (1996).

59. Donald, R. G. \& Roos, D. S. Stable molecular transformation of Toxoplasma gondii: a selectable dihydrofolate reductase-thymidylate synthase marker based on drug-resistance mutations in malaria. Proc. Natl Acad. Sci. USA 90, 11703-11707 (1993).

60. Donald, R. G. \& Roos, D. S. Insertional mutagenesis and marker rescue in a protozoan parasite: cloning of the uracil phosphoribosyltransferase locus from Toxoplasma gondii. Proc. Natl Acad. Sci. USA 92, 5749-5753 (1995).

61. Jacot, D., Daher, W. \& Soldati-Favre, D. Toxoplasma gondii myosin F, an essential motor for centrosomes positioning and apicoplast inheritance. EMBO J. 32, 1702-1716 (2013).

62. Krishnan, A. et al. Functional and computational genomics reveal unprecedented flexibility in stage-specific Toxoplasma metabolism. Cell Host Microbe 27, 290-306 e211 (2020).

63. Plattner, F. et al. Toxoplasma profilin is essential for host cell invasion and TLR11-dependent induction of an interleukin-12 response. Cell Host Microbe 3, 77-87 (2008).

64. Pino, P. et al. Mitochondrial translation in absence of local tRNA aminoacylation and methionyl tRNA Met formylation in Apicomplexa. Mol. Microbiol. 76, 706-718 (2010).

65. Agrawal, S., van Dooren, G. G., Beatty, W. L. \& Striepen, B. Genetic evidence that an endosymbiont-derived endoplasmic reticulum-associated protein degradation (ERAD) system functions in import of apicoplast proteins. J. Biol. Chem. 284, 33683-33691 (2009).

66. Ding, M., Clayton, C. \& Soldati, D. Toxoplasma gondii catalase: are there peroxisomes in toxoplasma? J. Cell Sci. 113(Pt 13), 2409-2419 (2000)

67. Mann, T. \& Beckers, C. Characterization of the subpellicular network, a filamentous membrane skeletal component in the parasite Toxoplasma gondii. Mol. Biochem Parasitol. 115, 257-268 (2001).

68. Herm-Gotz, A. et al. Toxoplasma gondii myosin A and its light chain: a fast, single-headed, plus-end-directed motor. EMBO J. 21, 2149-2158 (2002).

69. Rychlik, M. Mass spectrometric studies of trimethylsilylpantothenic acid and related substances. J. Mass Spectrom. 36, 555-562 (2001).

70. Zamboni, N., Fendt, S. M., Ruhl, M. \& Sauer, U. (13)C-based metabolic flux analysis. Nat. Protoc. 4, 878-892 (2009).

71. Adams, K. J. et al. Skyline for small molecules: a unifying software package for quantitative metabolomics. J. Proteome Res. 19, 1447-1458 (2020).

72. Clasquin, M. F., Melamud, E. \& Rabinowitz, J. D. LC-MS data processing with MAVEN: a metabolomic analysis and visualization engine. Curr. Protoc. Bioinforma. Chapter 14, Unit14 11 (2012).
73. Krishnan, A. Pantothenate biosynthesis is critical for the establishment of chronic infection by the neurotropic parasite Toxoplasma gondii. Mendeley Data, https://doi.org/10.17632/zyrz6dcc85.1 (2021).

74. Kloehn, J. Pantothenate biosynthesis is critical for chronic infection by the neurotropic parasite Toxoplasma gondii. Yareta Geneva Data Rep., https:// doi.org/10.26037/yareta:bvz6yrckafdrxmzgn5hpuumkue (2021).

75. Hehl, A. B. et al. Asexual expansion of Toxoplasma gondii merozoites is distinct from tachyzoites and entails expression of non-overlapping gene families to attach, invade, and replicate within feline enterocytes. BMC Genom. 16, 66 (2015)

\section{Acknowledgements}

This work was funded by the Swiss National Science Foundation (CRSII3_160702), and by the European Research Council (ERC) under the European Union's Horizon 2020 research and innovation program under grant agreement no. 695596. M.L. is supported by a PhD salary award granted by the Institute of Genetics and Genomics of Geneva (IGE3). We thank Dr. Pierre-Mehdi Hammoudi for the generation of the ME49 hxgprt-ko ku80-ko strain, Dr. Rebecca Oppenheim for the generation of the PPCDC-Ty tagged strain, Dr. Sunil Kumar Dogga for the support with the RNAseq analysis, and Remy Visentin (Protein platform University of Geneva) for assistance in protein purification. We thank Prof. Sebastian Lourido (Whitehead Institute) for sharing the ddBFD1 strain, and Alessandro Bonavoglia for help in characterizing it. We thank Eliane Sandmeier (MZ 2.0 facility) for setting up the GC-MS and Prof. Youssef Daali (University of Geneva) for the use of his QTRAP 6500 mass spectrometer. We finally thank Prof. Erick Strauss (Stellenbosch university) for critical reading and editing of the manuscript, as well as for the continuous discussion and input on the project.

\section{Author contributions}

D.S.F., M.L., J.K. and A.K. conceived the study; M.L., J.K., and O.V. designed, performed and interpreted the experimental work, with the support of A.K. and E.V.; D.S.F. supervised the research; M.L, J.K and D.S.F wrote the paper, with the support of O.V., A.K., and E.V.

\section{Competing interests}

The authors declare no competing interests.

\section{Additional information}

Supplementary information The online version contains supplementary material available at https://doi.org/10.1038/s41467-022-27996-4.

Correspondence and requests for materials should be addressed to Dominique SoldatiFavre.

Peer review information Nature Communications thanks Laura Knoll, Roberta Leonardi and the other, anonymous, reviewer(s) for their contribution to the peer review of this work. Peer reviewer reports are available.

Reprints and permission information is available at http://www.nature.com/reprints

Publisher's note Springer Nature remains neutral with regard to jurisdictional claims in published maps and institutional affiliations.

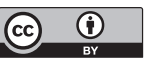

Open Access This article is licensed under a Creative Commons Attribution 4.0 International License, which permits use, sharing, adaptation, distribution and reproduction in any medium or format, as long as you give appropriate credit to the original author(s) and the source, provide a link to the Creative Commons license, and indicate if changes were made. The images or other third party material in this article are included in the article's Creative Commons license, unless indicated otherwise in a credit line to the material. If material is not included in the article's Creative Commons license and your intended use is not permitted by statutory regulation or exceeds the permitted use, you will need to obtain permission directly from the copyright holder. To view a copy of this license, visit http://creativecommons.org/ licenses/by/4.0/.

(C) The Author(s) 2022 